\title{
Trap-Related Reliability Problems of Dielectrics in Memory Cells
}

\author{
Hiroshi Watanabe *(D) and Hsin-Jyun Lin \\ Department of Electrical and Computer Engineering, National Yang Ming Chiao Tung University, \\ Hsinchu 30010, Taiwan; sinjyunlin.eed03g@nctu.edu.tw \\ * Correspondence: hwhpnabe@mail.nctu.edu.tw
}

check for

updates

Citation: Watanabe, H.; Lin, H.-J. Trap-Related Reliability Problems of Dielectrics in Memory Cells.

Electronics 2021, 10, 1287. https://

doi.org/10.3390/electronics10111287

Academic Editor: Tae-Yeon Seong

Received: 7 April 2021

Accepted: 25 May 2021

Published: 28 May 2021

Publisher's Note: MDPI stays neutral with regard to jurisdictional claims in published maps and institutional affiliations.

Copyright: (c) 2021 by the authors. Licensee MDPI, Basel, Switzerland. This article is an open access article distributed under the terms and conditions of the Creative Commons Attribution (CC BY) license (https:// creativecommons.org/licenses/by/ $4.0 /)$.

\begin{abstract}
A basic mechanism for storing data in memory cells is to record changes in electronic charges, material phases, resistivities, magnetic properties, and so forth. The change in electronic charge has been widely used in the majority of mass-produced memories, such as dynamic randomaccess memory (DRAM), static random-access memory (SRAM), NOR Flash, and NAND Flash. Other emerging memories have collected widespread attention for acquiring extra advantages which cannot be achieved using the change in electronic charge. Many years of studies have told us that reliability problems are critically important in the development of both conventional and emerging memories, in order to improve the product yield. However, the topics related to these problems are too wide to cover in these limited pages. In this review chapter, we address several interesting examples of trap-related problems in dielectrics for use in various memory cells. For engineering purposes, it is very important to grasp the relation of the achieved physical intuitions and electronic characteristics of dielectrics.
\end{abstract}

Keywords: dielectric; RTN; TAT; Wiener-Khinchin; transient analysis; phonon; surface roughness; spectral index; power spectrum

\section{Introduction}

There are two major innovations that human being have made since the appearance of mankind. One is the improvement of energy conversion efficiency. The other is the improvement of information communication efficiency. In the past few decades before 2020, semiconductor memory technologies have been regarded as belonging to the latter. However, as the energy that integrated circuit (IC) chips consume in data centers increases rapidly, the reduction of IC power consumption is also becoming meaningful for the global environmental problem. By multiplying the absolute temperature $(T)$ to Shannon's entropy, we can obtain the equality of energy and information quantity, where $p_{i}$ is the probability for state- $i$ (that is, $0 \leq p_{i} \leq 1$ for each $i$ ) and $k_{B}$ is the Boltzmann constant,

$$
|\Delta E|_{\text {min }}=-k_{B} T \sum_{i} p_{i} \operatorname{lnn} p_{i}
$$

During the period that we process information by $\sum_{i} p_{i} \ln p_{i}$ at $T$, we consume energy at least by $|\Delta E|_{\text {min }}$. By shortening this period following device scaling [1], we have so far improved the efficiency of information communication. However, this also tells us that the minimum energy that we consume per time unit, named the lower bound of power consumption, increases with the efficiency of information communication. If this continues, then the improvement in communication efficiency may surpass the improvement in energy conversion efficiency. From 2010 to 2020, the worldwide energy use of data centers stayed around 1\% [2]. However, in the next decade, there will be a risk of breaking the balance of efficiency improvement and the growth in energy use demands. This may be due to emerging applications in data intensive technologies such as artificial intelligence, 
automotive, and the internet-of-things. In response to this, memory technologies are being aggressively studied to reduce the power consumption of data centers [3]. From a similar viewpoint of power consumption, as well as big data accessibility [3], storage class memory is being extensively investigated [3-5]. In general, some part of the quantity of information must be noise in the wide range of electronic systems. The information entropy not related to the signal must be involved in the power consumption of memory systems. Therefore, basic research of the fluctuation sources caused by memory cells is critically important. In this chapter, we discuss electronic perturbations regarding dielectric films for use in memory cells.

\section{Electronic Perturbation Is Very Small and Discrete}

Following the law of large numbers, the average dominates fluctuations as the number of events increases. If the leakage current through a gate dielectric film is composed of tunneling events of electrons (depicted by the red and black arrows in Figure 1), then we can expect that the summed tunneling events turn out a continuous gate leakage current, as follows [6]: where $q$ is the elementary charge , $\hbar$ is Dirac's constant, $A$ is the gate area, $g(E)$ is the density-of-state for a given energy $E, D(E)$ is the tunneling probability, $f(E)$ is the Fermi distribution function, and $V_{g}$ is the gate voltage applied to the top electrode, while the bottom electrode is grounded,

$$
I=\frac{2 \pi q}{\hbar} \int d A \int d E g(E) D(E)\left(f\left(E+q V_{g}\right)-f(E)\right)
$$

In this equation, according to [6], the energy level in the cathode (bottom electrode) is higher by $q V_{g}$, because the energy level in the anode (top electrode) is lowered by $q V_{g}$. The Fermi distribution function is written as follows, where $\mu$ is the chemical potential,

$$
f(E)=\frac{1}{1+\exp \left(\frac{E-\mu}{k_{B} T}\right)} .
$$

In this figure, we assumed that there is an electronic perturbation, which is a local trap depicted by the yellow square. The trap-assisted tunneling may substantially enhance the tunneling, as depicted by the black bulk arrow. The red arrows depict the tunneling events without the trap-assistance. While the gate area $(A)$ is large, we can regard the number of tunneling events as very large. If the tunneling events without the trap-assistance prevail, then (2) is valid.

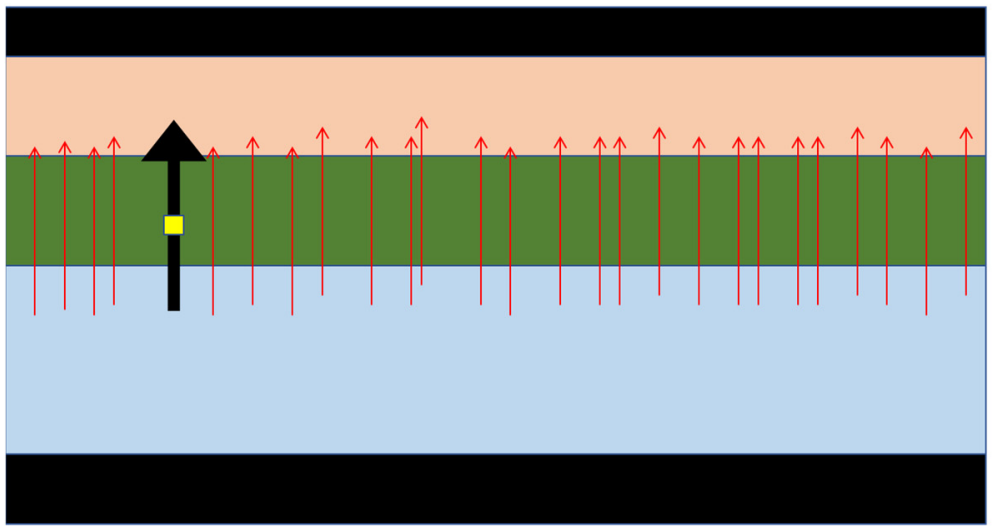

Top electrode

Gate metal

Dielectric film

Substrate (e.g., Si)

Bottom electrode

Figure 1. Continuum leakage [7]. The red arrows depict tunneling events with no trap-assistance. The black bulk arrow depicts tunneling event with trap-assistance. The yellow square depicts a local trap. The number of tunneling events with no trap-assistance prevails but the tunneling event is enhanced by the trap. As the gate area increases, the tunneling events with no trap-assistance dominate. 
However, in real engineering, we divide cells by etching, as illustrated in Figure 2. If most of cells have no local trap, then the trap-assisting can substantially increase the leakage only in the cell with a local trap. With device scaling, the geometry of the memory cells is shrunk so that the leakage through the cell with a local trap can be described by a discrete formula, as follows: where $M_{i, f}$ is the transition matrix element from the initial state- $i$ to the final state- $f$, and $E_{i}$ and $E_{f}$ are the energies of tunneling electrons at the initial state- $i$ and the final state- $f$, respectively,

$$
I=\sum_{i, f} \frac{2 \pi q}{\hbar}\left|M_{i, f}\right|^{2} \delta\left(E_{i}-E_{f}+\Delta E\right)
$$

The $E_{i}$ and $E_{f}$ correspond to the energy levels in the cathode and anode electrodes in (2), respectively. If $\Delta E \neq 0$, then it is an inelastic process with the energy loss being $\Delta E$. If $\Delta E=0$, then it is an elastic process. The $\Delta E$ is not the applied voltage.

What is important is that a smaller memory cell is more affected by a local event. Thus, it turns out to be easier to investigate a discrete electronic perturbation by using smaller memory cells than larger ones. For either two-dimensional (2D) or three-dimensional (3D) integration, with or without specific dielectric films, this is the common nature of the electronic perturbation. In order to investigate a discrete electronic perturbation by using a larger memory cell, a higher precision is necessary in the measurement. This, however, is usually difficult.

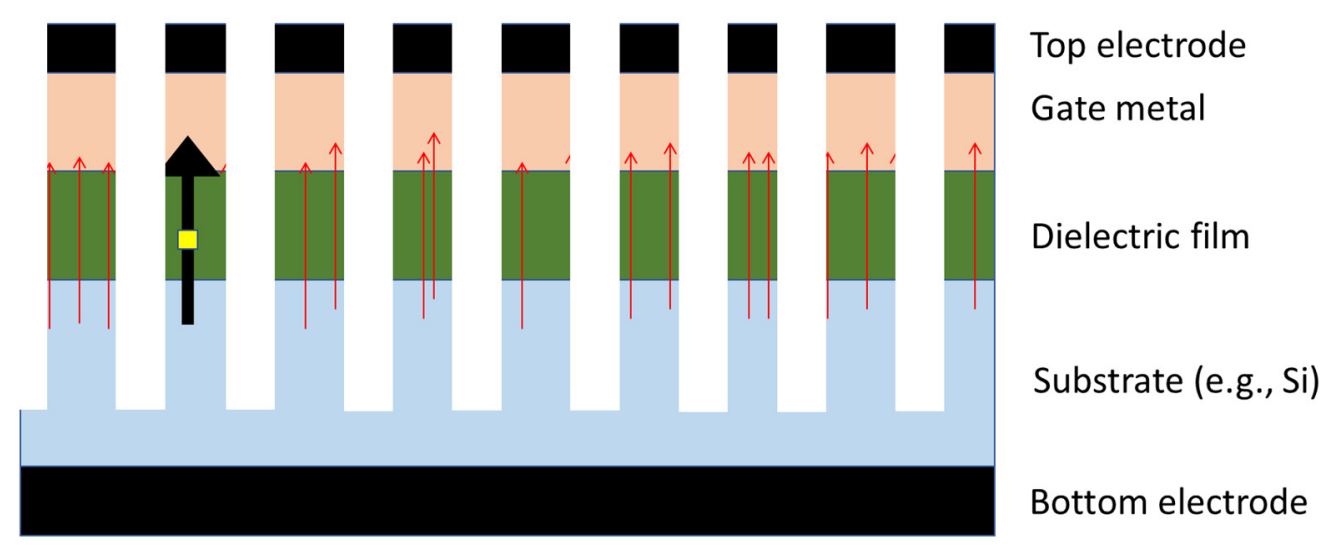

Figure 2. Discrete leakage [7]. After the etching, the capacitor is divided into many cells. The trap-assisted tunneling occurs through a cell having a local trap shows. The other cells are free from trap-assistance.

\section{Discrete Electronic Perturbation Is Unstable}

To carefully investigate a discrete electronic perturbation, a reliable oxide film is convenient for excluding non-targeted fluctuation factors, as far as possible. In [8], we prepared such a convenient sample using a $130 \mathrm{~nm}$ standard complementary metal-oxidesemiconductor (CMOS) process. Figure 3 contains an illustration and transmission electron microscope (TEM) images describing the sample geometry. The left-hand side (a) is a cross-sectional view along the cigar-shaped polysilicon above the substrate. The substrate surface is divided into two parts, labeled "S/D" and "G" by using the shallow trench isolation (STI). C1 is the capacitance between the cigar-shaped polysilicon and the substrate surface G. C2 is the capacitance between the cigar-shaped polysilicon and the substrate surface S/D. If the width of S/D is larger than that of $G$, then $C 2$ is greater than $C 1$, then the following capacitance coupling ratio $(C R)$ becomes greater than 0.5 .

$$
C R=\frac{C 2}{C 1+C 2}>0.5
$$


The center (b) is a bird's eye view of this sample. Along the perpendicular to (a), we have the source and drain diffusion layers, labeled " $\mathrm{S}$ " and " $\mathrm{D}$ ", respectively, on the substrate surface S/D. These diffusion layers sandwich the channel. We have two diffusion layers labeled " $\mathrm{G}$ " on the substrate surface $\mathrm{G}$. The diffusion layers, G, can connect to each other by the back-end of line (BEOL). The gate lengths on both substrate surfaces are the same as the width of the cigar-shaped polysilicon $(130 \mathrm{~nm})$. Thus, we can easily design $C R$ by tuning the gate width of both substrate surfaces (S/D and G). In this geometry, the gate oxide on the substrate surface $G$ can serve as a tunnel oxide, while $C R>0.5$. If a positive voltage is applied to the diffusion layers $S / D$, then the vertical electric field is concentrated above the substrate surface $\mathrm{G}$. When the voltage is large enough, an inversion layer is generated between the two diffusion layers $G$. Electrons coming from the diffusion layers $G$ to the inversion layer can be injected into the cigar-shaped polysilicon by Fowler-Nordheim tunneling (FNT).

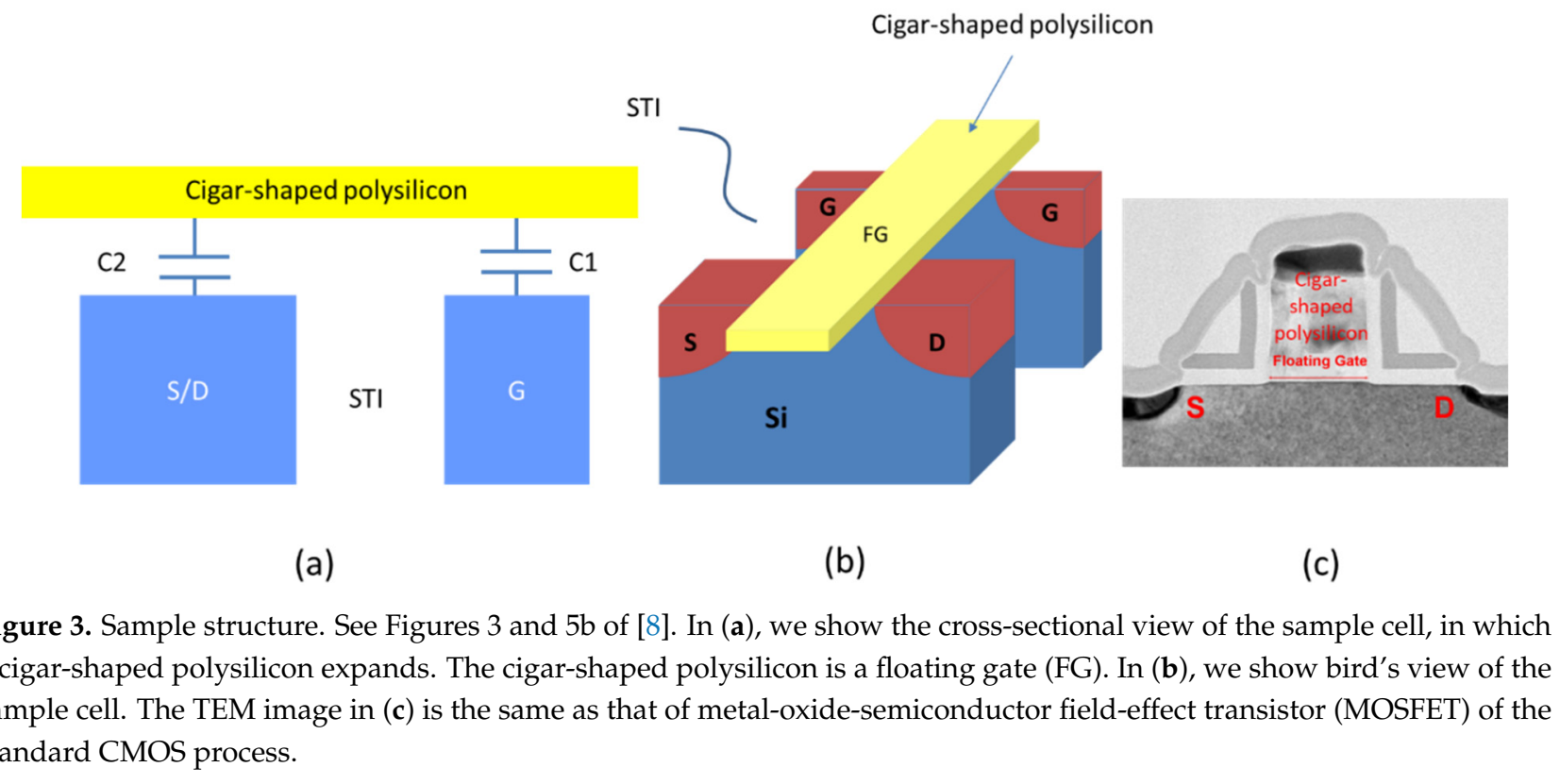

The righthand side image (c) is a TEM image of a cross-section in the plane perpendicular to the cigar-shaped polysilicon. It is the same as the cross-sectional view of the metal-oxide-semiconductor field effect transistor (MOSFET) of the $130 \mathrm{~nm}$ standard CMOS process, except for having no contact with the cigar-shaped polysilicon. Since the cigar-shaped polysilicon can in this way serve as the floating gate (FG), this sample is useful for various experiments of embedded type non-volatile memories (e.g., a battery-less timer [8]). Since the standard CMOS process is used to fabricate it, the quality of oxide and the substrate interface is within the scope of mass-production. Furthermore, we can obtain a sufficient number of sample cells in a mass-production line in which the cell-to-cell variation is under control.

We investigated the current flow of electrons from the diffusion layers, G to FG. The measurement scheme is as follows. We chose a sample cell with the gate lengths of $0.52 \mu \mathrm{m}$ and $0.4 \mu \mathrm{m}$ in the substrate surface $\mathrm{S} / \mathrm{D}$ and the substrate surface $\mathrm{G}$, respectively. This means that $C R=0.52 /(0.4+0.52) \cong 0.565$. We applied a voltage to the source and drain diffusion layers $S / D$, while the substrate and the diffusion layers $G$ were grounded. The substrate voltage was actually applied to the well contact, which is far from the channel between the diffusion layers S/D. Thus, we observed that $C R \times($ applied voltage $)-V t$ approximates the FG potential to the inversion layer being generated between the diffusion layers $\mathrm{G}$. While sweeping the voltage from zero to a voltage at which an abrupt increase appears (i.e., stopped by the ampere limiter), we measured the current flowing to the diffusion layers $G$ by subtracting the substrate current from it. This procedure was 
repeated multiple times in succession for each cell. However, between any two sequential measurements of each cell, the voltage became zero once. The breakdown voltage of a $130 \mathrm{~nm}$ node is about 7.2 V [9]. The repeated applications of nearly $7 \mathrm{~V}$ to the tunnel oxide may generate an electronic perturbation. The oxide thickness is $3 \mathrm{~nm}$ or less in a $130 \mathrm{~nm}$ node [9]. This is a little bit thinner than necessary to generate the FNT at $10 \mathrm{MV} / \mathrm{cm}$, which could cause the FNT in flash memories. The FNT is not caused by damage to the oxides. The tunnel gate area was about $0.052 \mu \mathrm{m}^{2}(=400 \mathrm{~nm} \times 130 \mathrm{~nm})$. The number of repetitions of voltage application was 30 for the measurement of each cell.

In Figure $4 \mathrm{a}$, we show the results of the first measurement of a chosen cell (along the vertical axis) over the electric field until 11.3 MV/cm (along the horizontal axis). The signal of the measured current was lower than the measurement limit. This limit was due to the equipment noise, and not of interest in this work. The leakage, if it exists, can be regarded as direct tunneling with no trap-assistance, that is, tunneling not assisted by traps (i.e., direct tunneling), as illustrated in Figure 4 b. Since we were interested in a discrete electronic perturbation in the oxide, we further continued the sequential measurement of this cell.

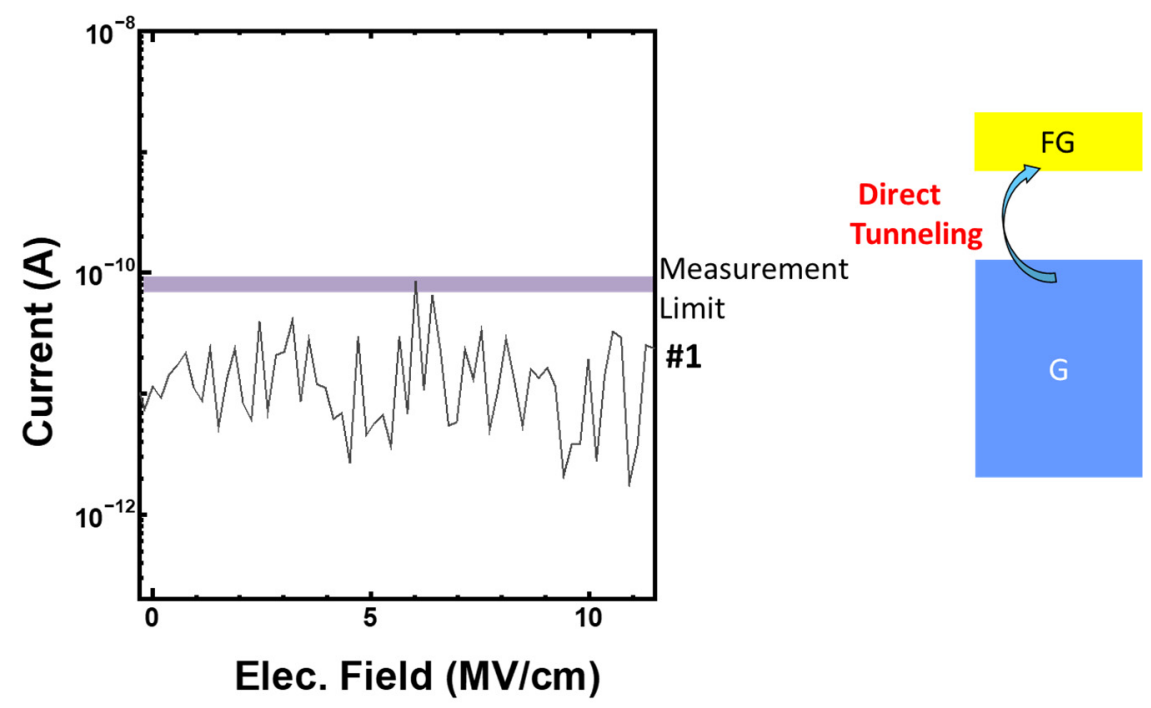

(a)

(b)

Figure 4. Experiments of stress-induced leakage current. See Figure 1 in [8]. In (a), the vertical axis is for the measured current and the horizontal axis is for the electric field applied to measure the current. The current obtained in the first measurement of the cell was smaller than the measurement limit. In (b), we illustrate the tunneling, which is tunneling not assisted by traps, that is, the direct tunneling. However, the direct tunneling is too small to be measurable with a small gate area.

In Figure 5a, we show the results of the 2nd to 18th measurements of the same cell as the first measurement. The vertical axis is for current and the horizontal is for electric field. A moderate increase of the current occurs from $5 \mathrm{MV} / \mathrm{cm}$. This moderate increase may be attributable to 1-trap process in a local trap having been generated in the previous measurement (stressing by more than $10 \mathrm{MV} / \mathrm{cm}$ ), as illustrated in Figure $5 \mathrm{~b}$. This is called the stress-induced leakage current (SILC) [10]. The leakage current is sensitive to the location and the energy level of the traps. Therefore, if the location and level of traps vary over the sequential measurement of the same cell, then the leakage current may vary in proportion to the fluctuation of the trap location and level. In (c), we consider the appearance of a second trap. If the location of the second trap is in a different tunnel path from that of the first trap, then it can increase the leakage current by about two-fold. Thus, the current of the 2nd to 18th measurements makes a bundle, as in Figure 5a. We, furthermore, continued the sequential measurement of the same cell. 


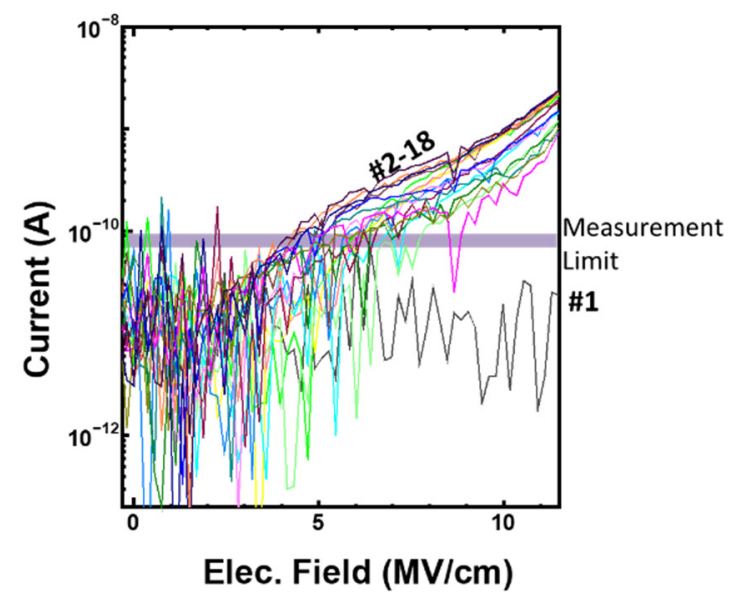

(a)

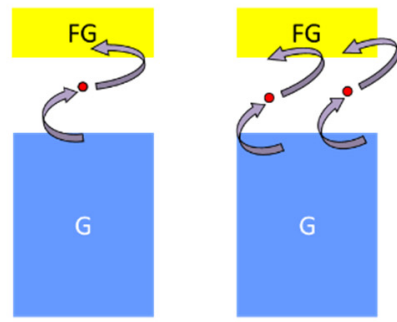

(b)

(c)

Figure 5. Experiments of stress-induced leakage current. See Figure 1 in [8]. In (a), the vertical axis is the measured current and the horizontal axis is the electric field applied to measure the current. The current obtained in the 2nd to 18th measurement of the same cell was larger than the measurement limit while the electric field was larger than $5 \mathrm{MV} / \mathrm{cm}$. In (b), trap-assisted tunneling is illustrated using a trap. In (c), there are two traps, which are in different tunnel paths from each other.

In Figure 6a, we show the results of the 19th and 20th measurements of the same cell (along the vertical axis) over the electric field (along the horizontal axis). There was a discontinuous increase from the moderate increase by ten-fold in the current level in the 19th and 20th measurements of the same cell from $2 \mathrm{MV} / \mathrm{cm}$. Ielmini et al. found that it is hard to explain this by using two traps which are in different tunnel paths [11]. Following them, we can regard this phenomenon as related to the two-trap process (i.e., two traps existing in a tunnel path [11]), as illustrated in Figure 6b. Accordingly, a second trap may be generated due to the stressing in the 18th measurement of the same cell. This implies that a local trap is a discrete perturbation. In (c), we can consider that there is another trap in a different tunnel path. However, the two-trap process may dominate it. We, furthermore, continued the sequential measurement of the same cell.

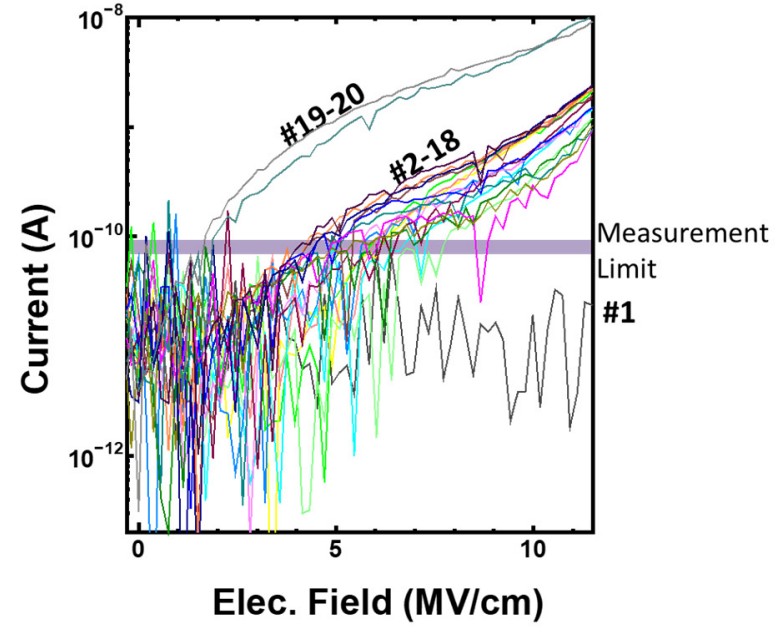

(a)

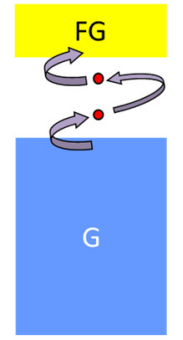

(b)

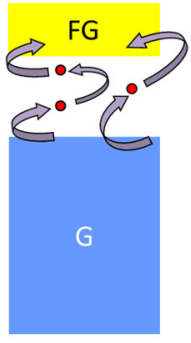

(c)

Figure 6. Experiments of stress-induced leakage current. See Figure 1 in [8]. In (a), the vertical axis is for the measured current and the horizontal axis is for the electric field applied to measure the current. The current obtained in the 19th and 20th measurement of the same cell was one-order larger than those obtained in the 2nd to 18th measurements of the same cell. In (b,c), we illustrate two-trap process for the mechanism for 19th to 20th measurement. 
In Figure 7a, we show the results of the 19th and 20th measurements of the same cell along the vertical axis over the electric field along the horizontal axis. What is surprising is that the current became lower than the measuring limit in the 21st to 30th measurements of the same cell. It is possible that the traps (i.e., discrete electronic perturbations) became inactivated or vanished. Therefore, we can regard the current in the 21st to 30th measurements as tunneling not assisted by traps (i.e., returning back to \#1), as illustrated in Figure $7 b$.

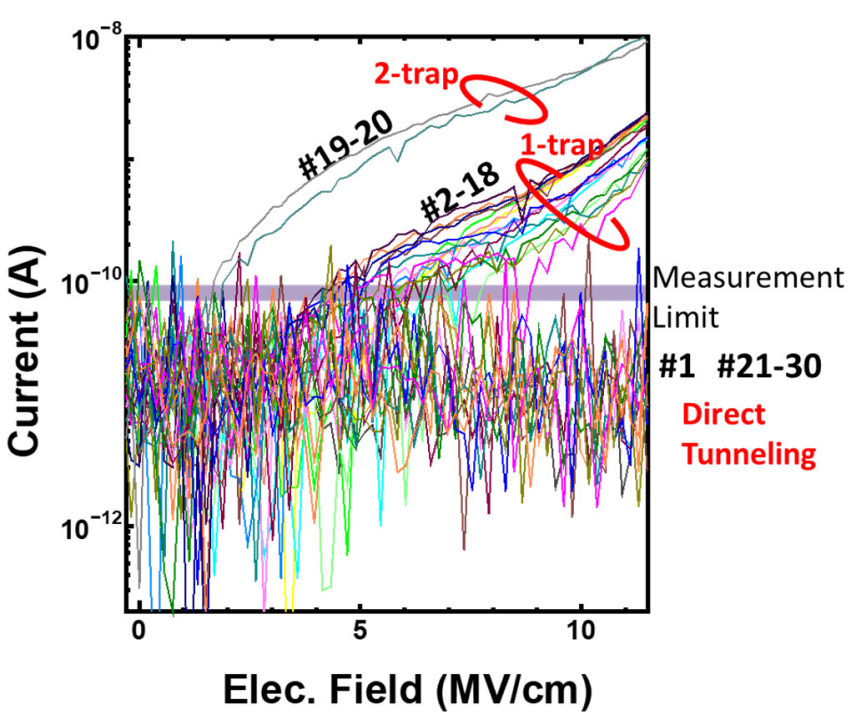

(a)

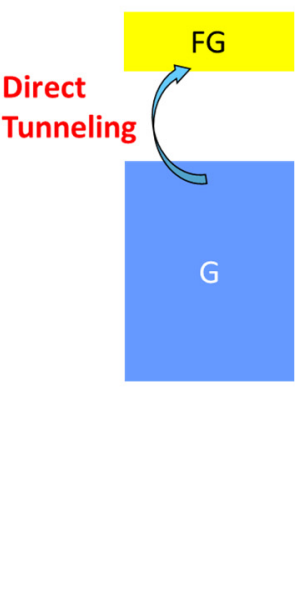

(b)

Figure 7. Experiments of stress-induce leakage current. See Figure 1 in [8]. In (a), the vertical axis is for the measured current and the horizontal axis is for the electric field applied to measure the current. The current obtained in the 21st to 30th measurements of the same cell was lower than the measurement limit. In (b), the mechanics for the 21st to 30th measurement goes back to the direct tunneling.

\section{What Is a Local Trap?}

This is a fairly difficult question because no one has observed (watched) one by a physical inspection method. As described in the above, the traps are unstable. It sometimes becomes activated, as shown in Figures $5 \mathrm{a}$ and $6 \mathrm{a}$. On another occasion, it becomes inactivated, as shown in the 21st to 30th measurements of the same cell in Figure 7a. Suppose that one tries to inspect a sample having shown a discrete increase in the leakage current; how can we acquire a spot to be clipped for obtaining a cross-section wherein a trap exists? Even if we could successfully know in which cross-section a trap exists, can unstable traps still exist after segmenting the cross-section? Or will another trap be generated by the segmentation of the cross-section? If someone succeeds in solving this problem, then he or she could win the Nobel prize.

The difficulty in the above can increase as the size of sample cells decreases, because the average number of traps decreases. If the gate area increases, then the number of traps may increase. Is it helpful to solve this problem? As mentioned above, a higher precision in the measurement is necessary if the gate area increases. In [12], the atto-ampere measurement is demonstrated. Such a method may be helpful for inspecting what is veiled under the measurement limit. It can be investigated if more trapping processes are hidden below the measurement limit [13]. If yes, the leakage process, which has been considered as tunneling not assisted by traps in Figure 7, could be further divided into several trapping processes. In this event, the one-trap and two-trap processes may turn out to be three-trap and four-trap processes, respectively, for example. Nevertheless, the basic research of such unstable and discrete electronic perturbations is critically important for memory device technologies. 


\section{Trap-Related Phenomena}

From experience, we have found that transient phenomena are more sensitive to discrete electronic perturbations. However, the phenomena due to discrete electronic perturbations are not always detectable. It may be observed occasionally while repeating the same measurement of the same cell, as mentioned above. In addition, if it is seen as an electric current change, it may accompany the continuous current with no relation to the electronic perturbations. A little ingenuity is necessary to study it.

There is a possible situation which is able to distinguish discrete electronic perturbations from a continuous current. In Figure 8, we illustrate stacked oxides with an interface layer (IL) sandwiched by two electrodes under a moderate electric field across the stacked oxides. The z-axis expands horizontally, and the conduction band edge is drawn on the vertical axis. The left electrode is the cathode that emits electrons to the right one (anode) due to direct tunneling (DT) along the $z$-axis. The IL expands over the X-Y plane perpendicular to the $z$-axis between the stacked oxides. In this exemplary illustration, the IL is composed of multiple interface traps having shallow trap levels. In (a), many electrons are stored in the IL, and thus the trap levels are piled up in the energy diagram. This increases the tunnel barrier and then suppresses the DT from the cathode to the anode, which is depicted by the red-dotted arrow. In this way, the tunnel barrier is modulated by stored charge in the traps [14]. The electric field across the oxide is enhanced on the anode side and weakened on the cathode side. The electrons likely are emitted from the IL to the anode in some way (e.g., Poole-Frenkel process [15]), while a sufficient amount of electrons are stored in the interface layer. In (b), the amount of electron charge in the IL was reduced as a result of the leakage, so that the trap levels became lower. The electric field was weakened on the anode side. In other words, the emission current reduces over time due to the emission itself, which is depicted by the red-bulk arrow. The transient current is composed of the emissions from the IL. The saturation current may be due to the DT with the reduced pile-up of the trap levels.

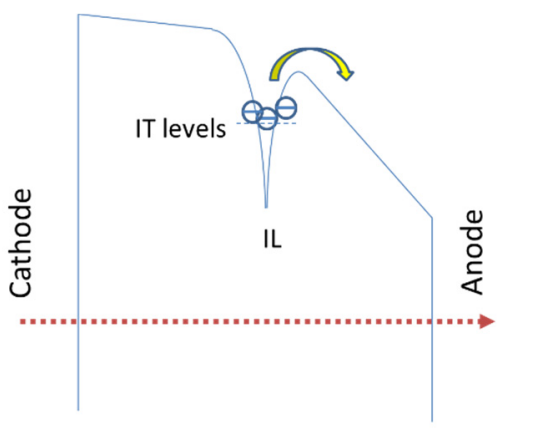

(a)

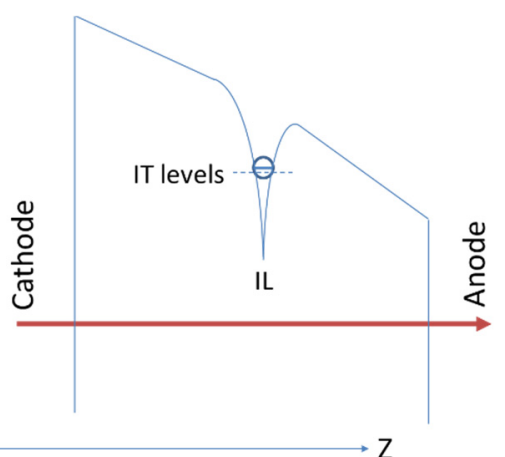

(b)

Figure 8. Emission and direct tunneling from an interface between the two oxide layers to be stacked. The horizontal axis depicts the z-axis, which is perpendicular to the interface (IL), and the energy band is drawn on the vertical axis. The IL has shallow trap levels, which are near to the oxide conduction band edge. In (a), the conduction band is piled up due to the charge of electrons captured in the IL. It suppresses the direct tunneling depicted by the red-dotted arrow. The Poole-Frenkel process may cause the leakage of the electrons from the IL. In (b), the reduction of electron charge in the IL recovers the direct tunneling depicted by the red-bulk arrow.

In Figure 9a, we can observe that the trap levels are lower than in Figure 8b. In such a case, trap-assisted tunneling (TAT) may likely occur. TAT is composed of the capture of electrons from the cathode by the IL due to DT, and the emission from the IL to the anode due to DT or FNT. The emission may be accompanied with energy loss if the TAT process is inelastic [16,17] (See $\Delta E$ in (2)). While the number of stored electrons in the IL is small, the emission is suppressed, and the capture is enhanced. When the number of stored electrons in the IL is large, the capture is suppressed, and the emission is enhanced. However, the 
emission may increase with the number of stored electrons and decrease with the decrease of stored electrons. This leads to a transient current due to the TAT. The saturation of TAT is achieved by the balance of the capture and the emission. The saturated TAT current, if it exists, must be much higher than the saturated DT current. The TAT can likely occur if the trap levels lower as the electric field increases.

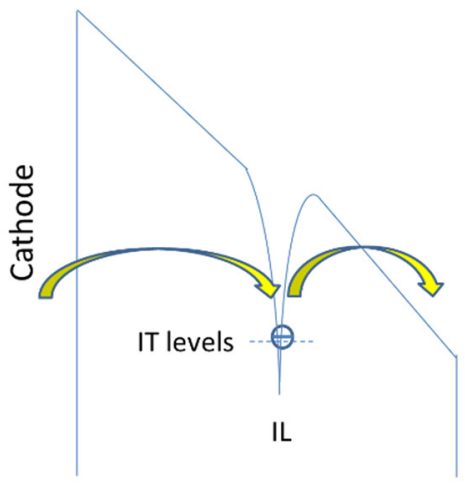

(a)

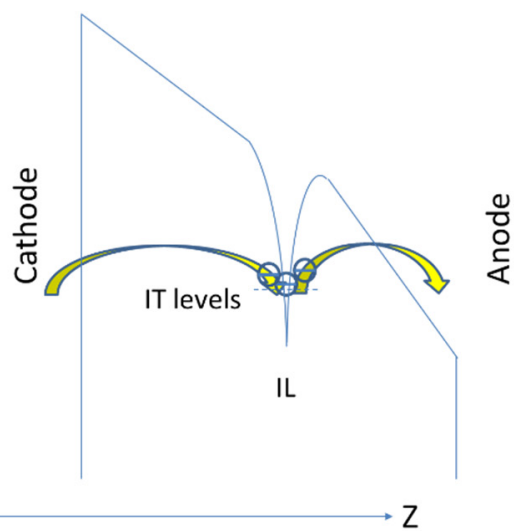

(b)

Figure 9. Trap-assisted tunneling (TAT). The z-axis expands horizontally, which is perpendicular to the interface (IL), and the conduction band edge is drawn in the vertical axis. The IL has a deeper trap level, which is far from the oxide conduction band edge. In (a), the IL has a smaller number of electrons. In (b), the IL has a larger number of electrons.

\section{Power Spectrum Analysis of Discrete Electronic Perturbations}

As we can understand from the discussion of Figures 8 and 9, the emission from the cathode or the IL is the key aspect that we have to analyze. The emission rate must be dependent of the electric field across the oxides, however, in this chapter, we a-priori assume that the emission rate is not explicitly dependent on time. The time dependence of the leakage current is assumed to be attributable only to the time-varying charge in the IL. In other words, we assume that the emission process is a stationary process. The autocorrelation of transient leakage current, $J_{g}(t) J_{g}{ }^{*}(t-u)$, can be thus regarded as independent of time $(t)$, where $u$ is the lag. We can depict it as $r_{J J}(u)$. We apply the Wiener-Khinchin theorem (6a) to analyze the power spectrum of the measured transient current, $S(f)$, where $f$ is the frequency, $E[\cdots]$ depicts the expectation value of $\cdots$ [18-20]. In (6b), $S(f)$ is proportional to $1 / f^{1+\alpha}$, where $1+\alpha$ is the spectral index, and ranges from 0.8 to $1.45[21,22]$. It was observed that the spectral index is sensitive to the location of electronic perturbations; at the interface or inside oxides [21,23].

$$
\begin{gathered}
S(f)=\int_{-\infty}^{\infty} E\left[r_{J J}(u)\right] \exp (-2 \pi i f u) d u \\
S(f) \sim f^{-(1+\alpha)}
\end{gathered}
$$

When $\alpha=0$, it is the $1 / f$-fluctuation, i.e., Flicker noise [24-28]. When $\alpha=1$, it is the $1 / f^{2}$-fluctuation, i.e., Brownian noise [29]. In general, the transient current varies over the samples to be measured. Therefore, it is indispensable to exclude the sample variations from the measured transient leakage current [30]. In Figure 10a, there is an illustration of the measured sample of a metal/high-K stack/metal (MIM) capacitor. The top and bottom electrodes are composed of metal (TiN). The top electrode is applied with a gate voltage $\left(V_{g}\right)$ to measure the current, while the bottom electrode is grounded. The high-K oxide stack is composed of a $5 \mathrm{~nm} \mathrm{TiO} 2$ layer and $10 \mathrm{~nm} \mathrm{ZrO}_{2}$ layer from the bottom. There may be an interface between the $10 \mathrm{~nm} \mathrm{ZrO}_{2}$ and the $5 \mathrm{~nm} \mathrm{TiO}$, which corresponds to the IL in Figures 8 and 9. The gate area is $2500 \mu \mathrm{m}^{2}$. The open probe fluctuation is about $10^{-10} \mathrm{~A}$, which is equivalent to the measuring limit in Figures $4-7$. Both the $10 \mathrm{~nm} \mathrm{ZrO}_{2}$ and the 
$5 \mathrm{~nm} \mathrm{TiO}_{2}$ are polycrystalline, as shown in the TEM image of Figure 10b. It was found that $\mathrm{ZrO}_{2}$ is cubic or tetragonal and $\mathrm{TiO}_{2}$ is rutile (tetragonal). Some of the grains inside the polycrystalline may face the IL. The distributions of grain orientations, grain sizes, and grain boundary geometry inside the oxide stack may cause the sample variations to be an obstacle for the power spectrum analysis.

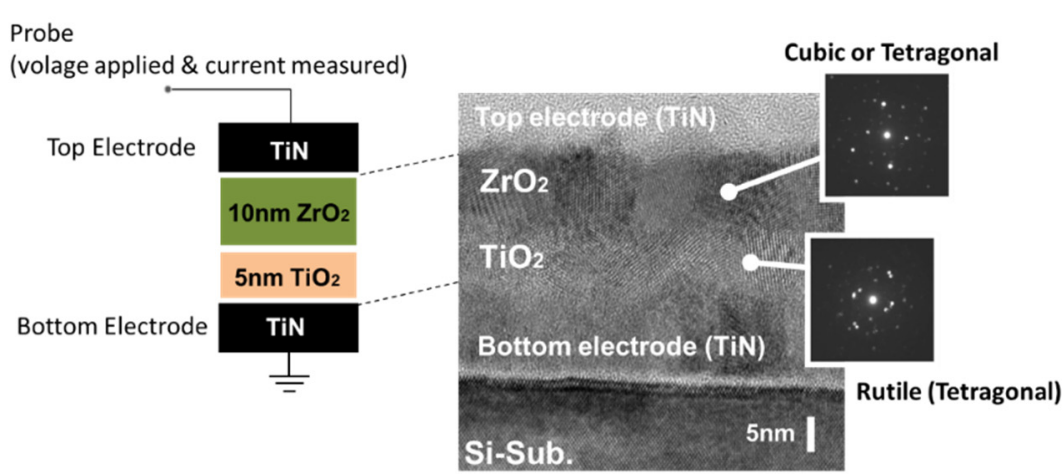

(a)

(b)

Figure 10. A sample of an MIM capacitor. See Figure 1 in [30]. The $z$-axis expands perpendicularly to the interface (IL) of $10 \mathrm{~nm} \mathrm{ZrO}_{2}$ having $\mathrm{K}=40$ and $5 \mathrm{~nm} \mathrm{TiO}{ }_{2}$ having $\mathrm{K}=130$. The bottom and top electrodes are TiN. In (a), the cross-sectional view is illustrated along the $z$-axis. In (b), the TEM image corresponding to (a) is shown. We can observe that both $\mathrm{ZrO}_{2}$ and $\mathrm{TiO}_{2}$ are polycrystalline.

In Figure 11a,b, we plotted the transient current measured using nine samples (labeled $\# 1-\# 9)$ having the same geometry as Figure 11a. The vertical axis is the measured current density and the horizontal is time. In (a), the transient current of the samples was measured under a positive fixed electric field $(+0.5 \mathrm{MV} / \mathrm{cm})$. In $(\mathrm{b})$, the transient current of the samples was measured under a negative fixed electric field $(-0.5 \mathrm{MV} / \mathrm{cm})$. First of all, the plot shows that the sample-to-sample variation (named, the sample variation) was quite large. Next, we can see an uneven fluctuation (discrete electronic perturbations) in the data of the sample \#1 under $-0.5 \mathrm{MV} / \mathrm{cm}$ and so forth.

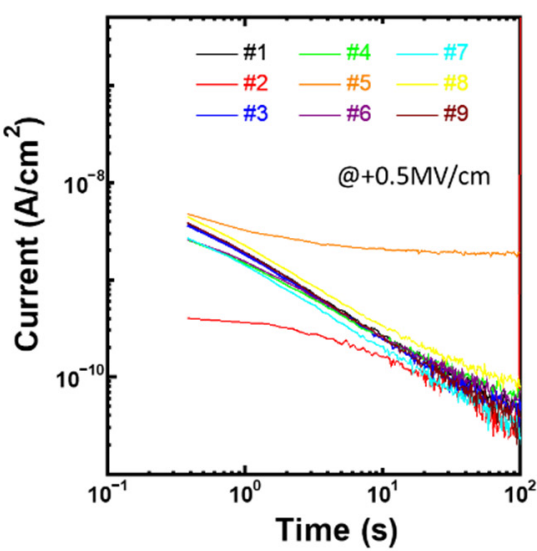

(a)

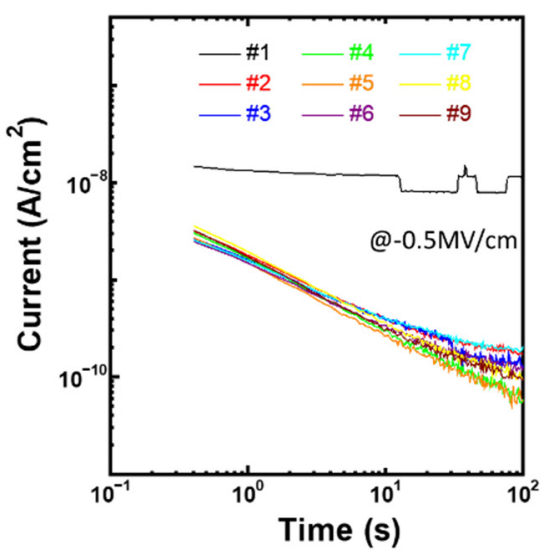

(b)

Figure 11. Measured transient leakage current using nine samples under $+0.5 \mathrm{MV} / \mathrm{cm}$ and $-0.5 \mathrm{MV} / \mathrm{cm}$ in $(\mathbf{a}, \mathbf{b})$, respectively. See Figure 2 in [30]. The vertical axis is the measured current density, and the horizontal is time. In (b), we have an uneven component (slow fluctuation due to electronic perturbation) in the sample \#1. We can distinguish the measured data with the uneven component from those without the uneven component. 
To analyze the electronic perturbation appropriately, we have to exclude the sample variation. In Figure 12, we illustrate the band diagram of Figure 10 with a moderate positive $V_{g}$ applied, which corresponds to $+0.5 \mathrm{MV} / \mathrm{cm}$. The horizontal axis is the $z$-axis (depth direction) and the vertical axis is the conduction band edge of the stacking film sandwiched by the electrodes. From the bottom, there are three interface layers (IL-1, IL-2, and IL-3) at the interfaces between the bottom electrode (TiN) and the $5 \mathrm{~nm} \mathrm{TiO}_{2}$, between the $5 \mathrm{~nm} \mathrm{TiO}{ }_{2}$ and the $10 \mathrm{~nm} \mathrm{ZrO}_{2}$, and between the $10 \mathrm{~nm} \mathrm{ZrO}_{2}$ and the top electrode (TiN), respectively. In this figure, we assumed no electrons were stored in the interface layers (i.e., the IL-1, the IL-2, and the IL-3). The barrier height of the $5 \mathrm{~nm} \mathrm{TiO} 2$ is $0.35 \mathrm{eV}$ from the bottom electrode [31-34]. The barrier height of the $10 \mathrm{~nm} \mathrm{ZrO}_{2}$ from the top electrode is $0.94 \mathrm{eV}$ [31-34]. In general, the dielectric constant decreases with the band gap (or tunnel barrier). Therefore, the electric field across the $10 \mathrm{~nm} \mathrm{ZrO}$ is higher than the $5 \mathrm{~nm} \mathrm{TiO}$, while we can ignore the stored electrons in the interface layers.

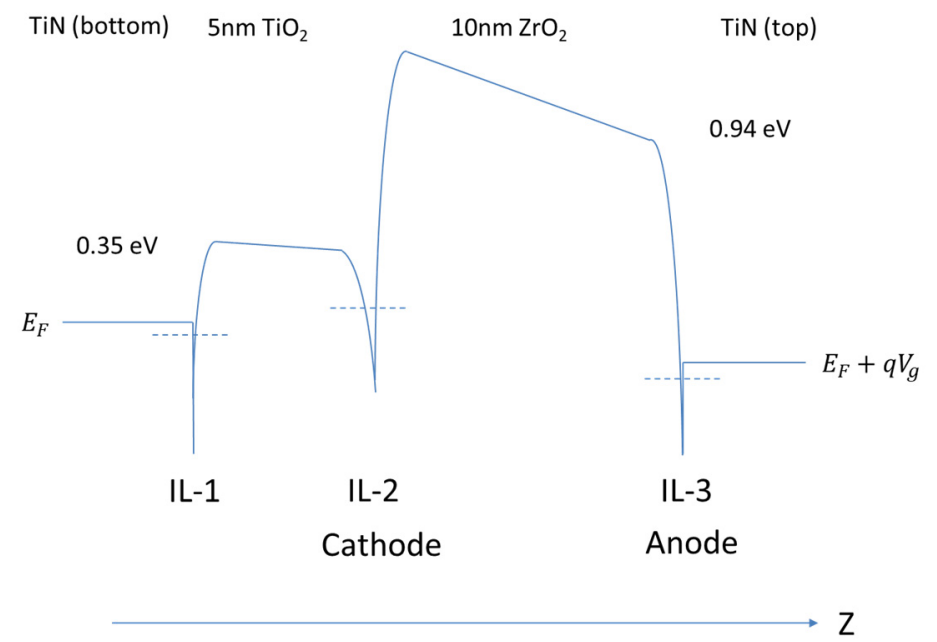

Figure 12. Band diagram with no electrons in the interface layers (IL-1, IL-2, IL-3) under a positive electric field. The horizontal axis is along the $z$-axis (depth direction) and the vertical axis is the conduction band edge of the stacking film sandwiched by the electrodes. There are three interface layers. Among them, IL-2 and IL-3 sandwich the $\mathrm{ZrO}_{2}$, which dominates the tunnel resistance.

In a usual analysis, the IL-1 and IL-3 may be ignored by supposing that the electrons come from an electrode to the other electrode passing through homogenous oxides (having no sample variations). However, both the oxide stack and the interface layers must have sample variations due to grain-related distributions. In other words, for $+0.5 \mathrm{MV} / \mathrm{cm}$, an electron can be emitted from the IL- 1 to the IL-2 through the $5 \mathrm{~nm} \mathrm{TiO}{ }_{2}$ with sample variations and then be emitted to the IL-3 through the $10 \mathrm{~nm} \mathrm{ZrO}_{2}$ with sample variations. Due to the higher barrier and the thicker width, the $10 \mathrm{~nm} \mathrm{ZrO}_{2}$ dominates the serial resistance of this oxide stack. Therefore, for a positive electric field, to analyze the transient current, we must consider the emission of electrons from the IL-2 (i.e., the cathode interface) to the IL-3 (i.e., the anode interface). For a negative electric field, we must consider the emission of electrons from the IL-3 (i.e., the cathode interface) to the IL-2 (i.e., the anode interface). That is, the IL-2 and IL-3 are the cathode interfaces for a positive and negative electric field, respectively. The IL-2 and IL-3 are the anode interfaces for a negative and positive electric field, respectively. The DT may likely occur between the IL-2 and the IL-3 due to the high tunnel barrier under a moderate electric field. Thus, we have the sample variations (see Figure 11) that may be attributable to the distribution of grains inside the $10 \mathrm{~nm} \mathrm{ZrO}_{2}$ sandwiched by the IL-2 and IL-3. If we have a sufficient number of stored electrons in the IL-2, the band diagram is modulated to Figure 13. This corresponds to Figure 9b. 


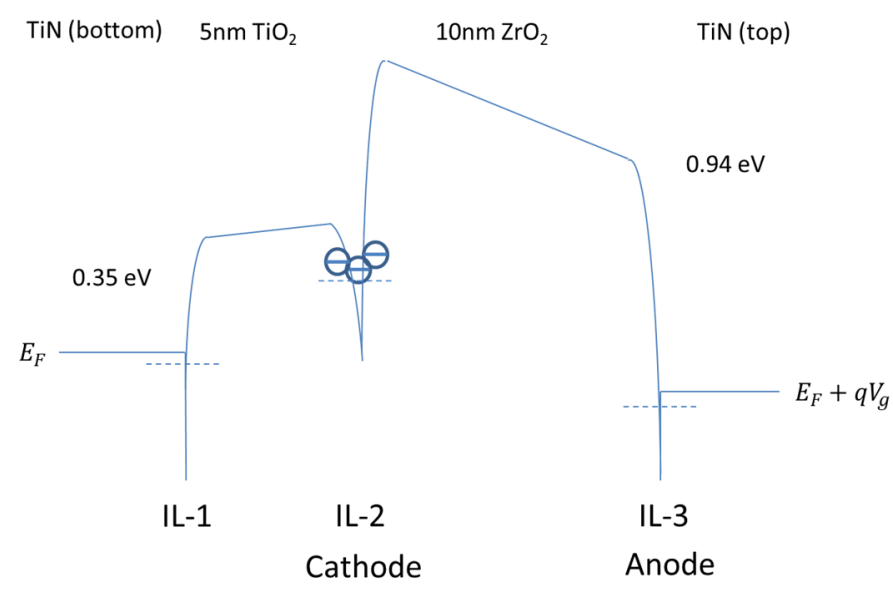

Figure 13. Band diagram with sufficient electrons stored in the cathode under a positive electric field. The horizontal axis is along the $z$-axis (depth direction) and the vertical axis is for the conduction band edge of the stacking film sandwiched by the electrodes.

The $10 \mathrm{~nm} \mathrm{ZrO}_{2}$ is a polycrystalline having variations of grain orientation, grain sizes, and the grain boundary patterns inside. The IL-2 and IL-3 may also be affected by the orientations, the sizes, and the boundary patterns of the grains facing the interfaces. This may be the source of the sample variations. In [30], we regulated these complex sources of sample variation and then obtained a formula to analyze the transient DT current density, as follows: where $J_{g, \infty}$ is the stationary DT current density between the IL-2 and the IL-3.

$$
J_{D T}(t) \approx J_{g, \infty}\left(1-\operatorname{sexp}\left(-\frac{t}{\tau}\right)\right)^{-1}
$$

We, a-priori, ignore the influence of the anode interface on the sample variations and then consider the grain-related variations in the cathode interface and the $10 \mathrm{~nm} \mathrm{ZrO}_{2}$. However, $s$ is the average of surface roughness, which varies over grains facing the cathode interface, and $\tau$ is the average of the longest dwell time of electrons which are emitted from the cathode interface over the grains. The $s$ is always positive and less than unity. As $s$ is close to unity, the surface roughness reduces at the cathode interface.

Subsequently, suppose there is a trapping site inside the $10 \mathrm{~nm} \mathrm{ZrO}_{2}$, as illustrated in Figure 14. The horizontal axis is the $z$-axis (depth direction) and the vertical axis is the conduction band edge of the stacking film sandwiched by the electrodes. If the energy levels of the trapping sites are low enough, then the TAT may likely occur from the cathode interface (IL-2) to the anode interface (IL-3) via the trapping site. This may dominate the direct tunneling between the IL-2 and the IL-3. In a similar way to the derivation of (7), we obtained the formula to analyze the transient TAT current density, as follows: where $J_{g, \infty}^{\prime}$ is the stationary TAT current density, $\tau^{\prime}$ is the dwell time of electrons emitted from the trapping sites for the TAT process, and $s^{\prime}$ is a positive number.

$$
J_{T A T}(t) \approx J_{g, \infty}^{\prime}\left(1-s^{\prime} \exp \left(-\frac{t}{\tau^{\prime}}\right)\right)^{-1}
$$

However, the trapping sites may also vary in depth in the energy diagram between samples. In one case, the electrons are stuck in the trapping sites. This cannot contribute to the measured leakage current. If $s^{\prime}$ is smaller than unity, the TAT dominates. Otherwise, the electron is stuck as a fixed charge there. 


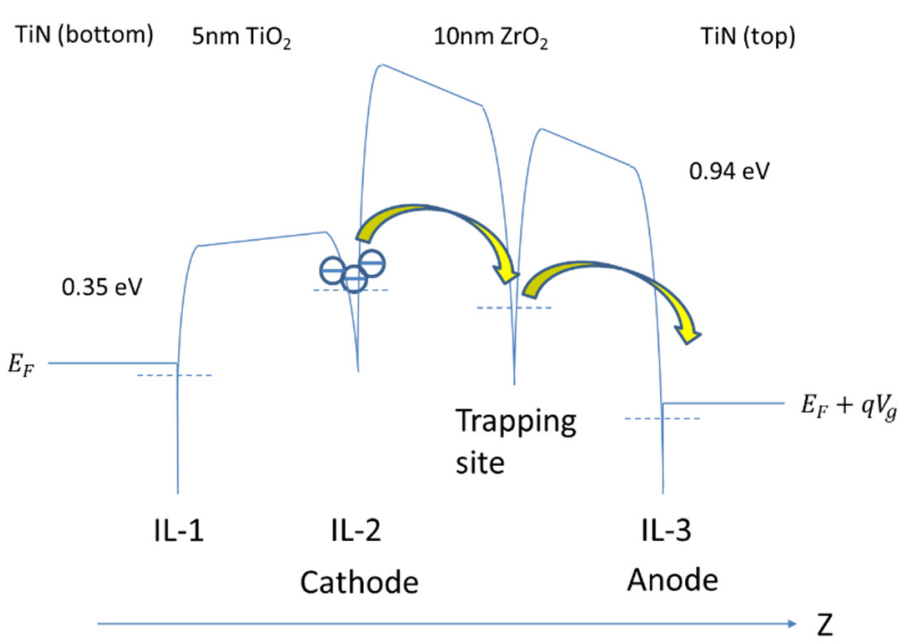

Figure 14. Band diagram with a trapping site inside the $10 \mathrm{~nm} \mathrm{ZrO}_{2}$ under a positive electric field. The horizontal axis is along the $z$-axis (depth direction) and the vertical axis is the conduction band edge of the stacking film sandwiched by the electrodes.

The parameters $\left(J_{g, \infty}, \tau, s, J_{g, \infty}^{\prime}, \tau^{\prime}, s^{\prime}\right)$ characterize the sample variations and not the discrete electronic perturbations. By tuning these parameters, we obtained an excellent agreement with the measured data, without uneven fluctuations (i.e., discrete electronic perturbations). An example is sample No. 6 (\#6), shown in Figure 15. In (a), the vertical axis is the current density and the horizontal one is time. The measured data under the electric fields $(0.5 \mathrm{MV} / \mathrm{cm}, 0.7 \mathrm{MV} / \mathrm{cm}$, and $1 \mathrm{MV} / \mathrm{cm})$ are depicted by black open circles. The lowest current can be excellently reproduced only by using (7) (i.e., DT only). The others can be excellently reproduced by using both (7) and (8) (i.e., both DT and TAT). The TAT can become involved as the electric field increases. In (b), the vertical axis is for the power spectrum densities and the horizontal one is for frequency. The black, red and blue open circles depict the power spectrum densities converted from the measured transient current densities at $+0.5 \mathrm{MV} / \mathrm{cm},+0.7 \mathrm{MV} / \mathrm{cm}$, and $+1.0 \mathrm{MV} / \mathrm{cm}$ using (6a), respectively. The calibrated $\alpha$ is $0.224,0.321$, and 0.408 at $+0.5 \mathrm{MV} / \mathrm{cm},+0.7 \mathrm{MV} / \mathrm{cm}$, and $+1.0 \mathrm{MV} / \mathrm{cm}$ using (6b), respectively. It increases as TAT becomes involved. The calibrated parameters are shown in Table 1. In Figure 16a, the measured transient current of samples No. 2 (\#2), No. 6 (\#6), and No. 8 (\#8) under $+0.5 \mathrm{MV} / \mathrm{cm}$ are depicted by black open circles. The vertical axis is the current density and the horizontal one is time. We obtained an excellent agreement by using only (7) (i.e., DT). In (b), the vertical axis is the power spectrum densities and the horizontal one is frequency. The red, black, and blue open circles depict the power spectrum densities converted from the measured transient current densities of samples \#2, $\# 6$, and \#8 at $+0.5 \mathrm{MV} / \mathrm{cm}$ using (6a), respectively. The black open circles are the same as in Figure 15b. In addition, we observed that $\alpha=0.813$ and 0.108 for $\# 2$ and $\# 8$ at $+0.5 \mathrm{MV} / \mathrm{cm}$ using (6b), respectively. The calibrated parameters are also shown in Table 1.

Table 1. Calibrated fitting parameters for Figures 15 and 16. See Table 1 in [30]. In the lowest electric field (+0.5 MV/cm), the leakage current is dominated by the direct tunneling (DT). When increasing the electric field, trap-assisted tunneling (TAT) can become involved.

\begin{tabular}{|c|c|c|c|c|c|c|c|c|c|}
\hline Sample No. & $\begin{array}{c}\text { Field } \\
(\mathrm{MV} / \mathrm{cm})\end{array}$ & $\begin{array}{c}J_{g, \infty} \\
\left(\mathrm{pA} / \mathrm{cm}^{2}\right)\end{array}$ & $\tau(s)$ & $s$ & $\begin{array}{c}J_{g, \infty}^{\prime} \\
\left(\mathrm{pA} / \mathrm{cm}^{2}\right)\end{array}$ & $\tau^{\prime}(\mathrm{s})$ & $s^{\prime}$ & Note & $\alpha$ \\
\hline$\# 2$ & 0.5 & 17.5 & 120 & 0.9580 & $\mathrm{~N} / \mathrm{A}$ & $\mathrm{N} / \mathrm{A}$ & $\mathrm{N} / \mathrm{A}$ & DT & 0.813 \\
\hline \multirow{3}{*}{ \#6 } & 0.5 & 51 & 48 & 0.9885 & $\mathrm{~N} / \mathrm{A}$ & $\mathrm{N} / \mathrm{A}$ & $\mathrm{N} / \mathrm{A}$ & DT & 0.224 \\
\hline & 0.7 & 56 & 75 & 0.9962 & 390 & 0.5 & 0.5 & $\mathrm{DT}+\mathrm{TAT}$ & 0.321 \\
\hline & 1.0 & 140 & 70 & 0.9920 & 860 & 0.1 & 0.1 & $\mathrm{DT}+\mathrm{TAT}$ & 0.408 \\
\hline$\# 8$ & 0.5 & 87.5 & 32 & 0.9920 & $\mathrm{~N} / \mathrm{A}$ & $\mathrm{N} / \mathrm{A}$ & $\mathrm{N} / \mathrm{A}$ & DT & 0.108 \\
\hline
\end{tabular}




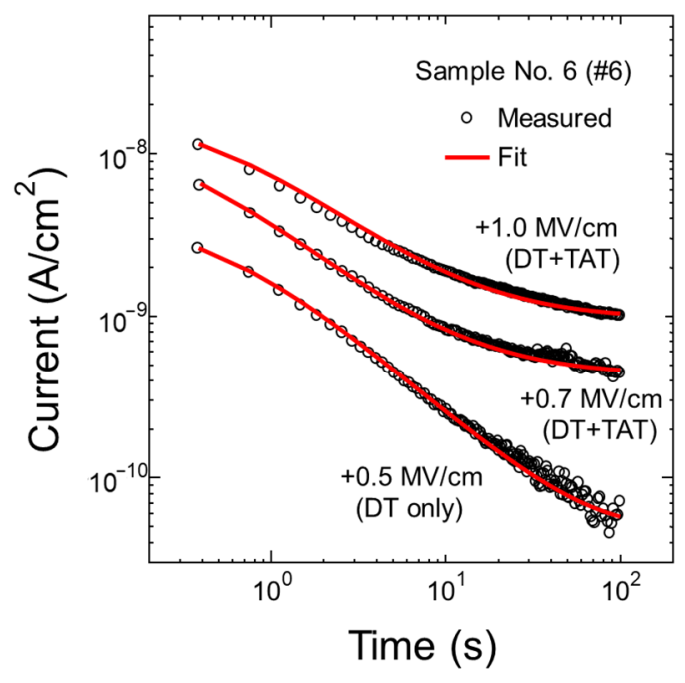

(a)

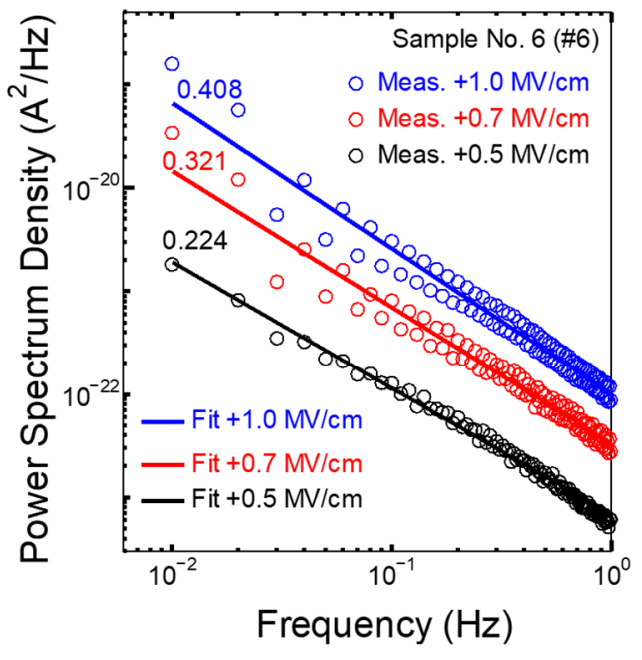

(b)

Figure 15. Fitting of the sample No. 6 (\#6) under moderate electric fields $(+0.5,+0.7$, and $+1.0 \mathrm{MV} / \mathrm{cm})$. See Figure 5 in [30]. In (a), the vertical axis is the current density and the horizontal one is time. The marks depict the measured current and the red lines depict the fit using (7) and/or (7). In (b), the marks depict the power spectrum densities converted from the measurement using (6a). The lines fit the measurement for each electric field using (6b) and then we obtained $\alpha=0.224,0.321$, and 0.408 for $+0.5 \mathrm{MV} / \mathrm{cm},+0.7 \mathrm{MV} / \mathrm{cm}$, and $+1.0 \mathrm{MV} / \mathrm{cm}$, respectively.

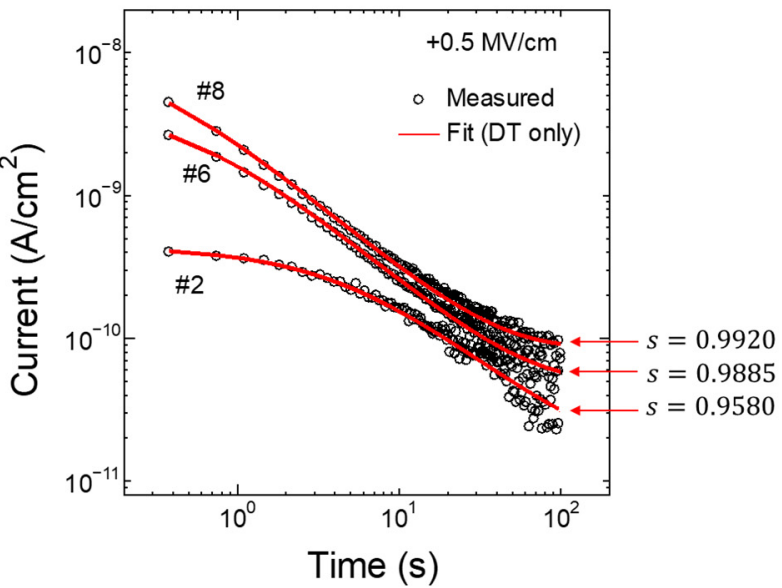

(a)

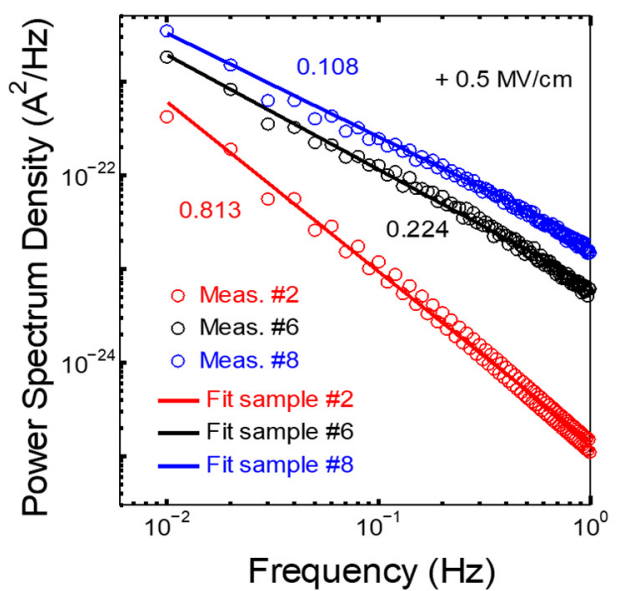

(b)

Figure 16. Fitting of the transient direct tunnel current. See Figure 6 in [30]. In (a), the vertical axis is the current density and the horizontal one is time. The marks depict the measured transient leakage current densities using sample \#2, \#6, and \#8. The red lines depict the fit using (6a) for each sample. The calibrated $s$ is $0.9580,0.9885$, and 0992 for \#2, \#6, and $\# 8$, respectively. In (b), the vertical axis is the power spectrum distribution and the horizontal one is the frequency. The marks were converted from measured data using (61). The red line is to fit $\alpha$ using (6b). The calibrated $\alpha$ is $0.813,0.224$, and 0.108 , respectively.

In Figure 17, the vertical axis is $\alpha$ and the horizontal is the surface roughness index (s). The open dots depict those calibrated using sample \#6 at $+0.5 \mathrm{MV} / \mathrm{cm},+0.7 \mathrm{MV} / \mathrm{cm}$, and $+1.0 \mathrm{MV} / \mathrm{cm}$ in Figure 16a,b, respectively. Since these transient currents can only be fit using (7) (i.e., DT only), the number of fitting parameters is three $\left(J_{g}, \tau\right.$, and $\left.s\right)$. See Table 1. Let us choose $J_{g, \infty}=51 \mathrm{pA} / \mathrm{cm}^{2}$ and $\tau=48 \mathrm{~s}$ from the sample $\# 6$ at $+0.5 \mathrm{MV} / \mathrm{cm}$ in Table 1 (fit by DT). Regarding $s$ as a variable, we can calculate the transient DT current using (7). Substituting it for (6a), we can calibrate $\alpha$ using (6b) for a given $s$, as plotted 
using the line in Figure 17. It is thus found that $\alpha$ increases as $s$ decreases from unity (i.e., no surface roughness). We can thus find that the surface roughness violates the power law of the Flicker noise $(\alpha=0)$. See Table 1 again. In sample $\# 2$ at $+0.5 \mathrm{MV} / \mathrm{cm}$, the transient current was reproduced using only (7) (i.e., only DT) and $\alpha=0.813$, which is very high. However, $s=0.9580$, which is very small compared with the others. This means that \#2 has a larger surface roughness. According to the trend in Figure 17, we can assume that $\alpha$ would reduce from 0.813 , as the surface roughness could have been suppressed in sample $\# 2$. If we can reduce the surface roughness, then $\alpha$ may also have decreased in samples \#6 and \#8 at $+0.5 \mathrm{MV} / \mathrm{cm}$ from 0.224 and 0.108 , respectively. Thus, we can observe that $\alpha \cong 0$ (i.e., the spectral index is 1) when the electrons are directly emitted from the cathode to the anode interfaces (i.e., DT). The DT turns out the Flicker noise and the surface roughness violates the power of the Flicker noise. In sample \#6, furthermore, $\alpha$ increases due to the TAT as the electric field increases. The TAT also violates the power law of the Flicker noise.

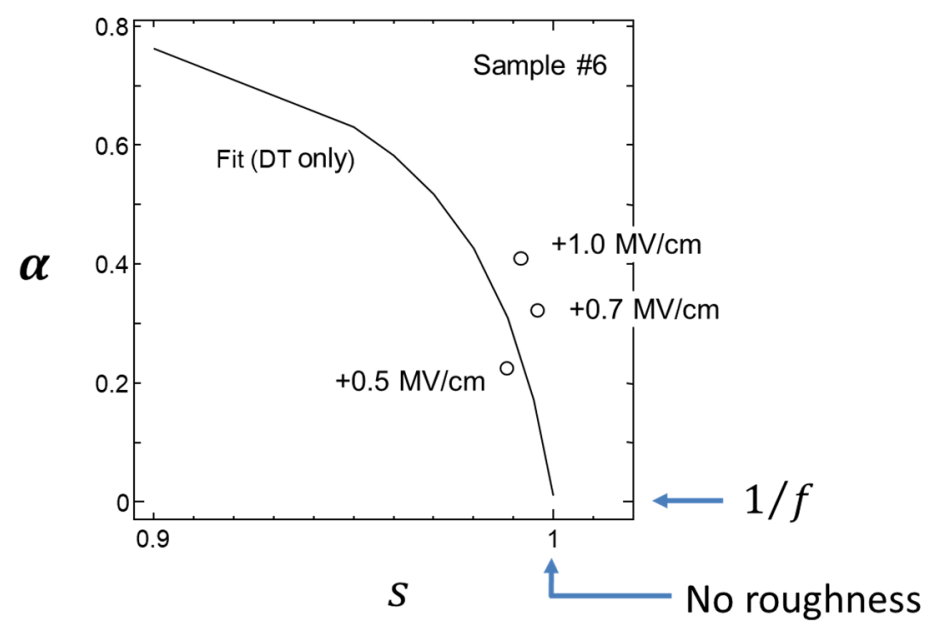

Figure 17. Impact of surface roughness. See Figure 7a in [30]. The parameters ( $\alpha$ and $s$ ) obtained by fitting the measurement are plotted using a mark for the sample \#6 at $+0.5 \mathrm{MV} / \mathrm{cm},+0.7 \mathrm{MV} / \mathrm{cm}$, and $+1.0 \mathrm{MV} / \mathrm{cm}$. The line depicts the theoretical prediction using $J_{g, \infty}$ and $\tau$, calibrated to fit the measured transient current for sample \#6 at $+0.5 \mathrm{MV} / \mathrm{cm}$. The surface roughness violates the power law of the Flicker noise so that $\alpha$ increases as $s$ decreases.

As mentioned above, we can observe a fluctuation which may be discrete and subject to neither (7) nor (8). For example, there is an uneven transient current for sample No. 1 (\#1) under $-0.5 \mathrm{MV} / \mathrm{cm}$ in Figure 11b. This measured data is replotted using lines and dots in Figure 18a. The vertical axis is the current density and the horizontal one is time. There are three discrete perturbations. In (b) of this figure, by tuning the fitting parameters of (7) and (8), we can obtain the continuous transient current depicted by the red dash line, with the calibrated fitting parameters shown in Table 2. By subtracting it from the measured transient current (depicted dot and line), we obtained the red line. It appears as the summation of a stationary current $\left(\cong 10^{-8} \mathrm{~A}\right)$ and the uneven component of the fluctuating current with no continuous decay. The calibration of the parameters was carried out to exclude the continuous decay from the red line. That is, we can deduce the uneven component from the measured data. We considered the uneven components as randomtelegraph noise (RTN) in the transient leakage current [35]. Machlup assumed bi-states (i.e., discrete) and used (6a) and (6b) to study RTN [18]. Its origin has been considered to be related to number fluctuation [24], to mobility fluctuation [36-38], to both number and mobility fluctuations [39-41], and phonon scattering [42]. 


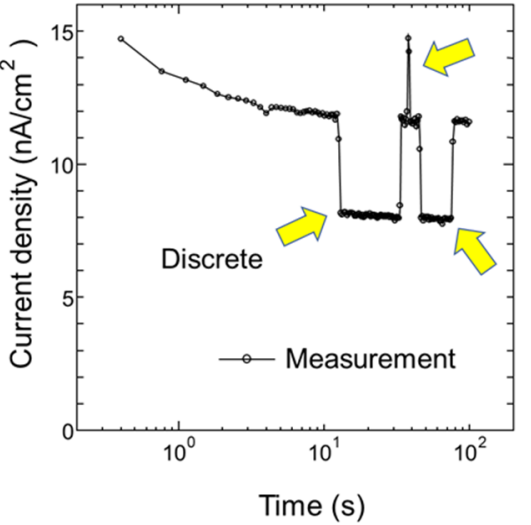

(a)

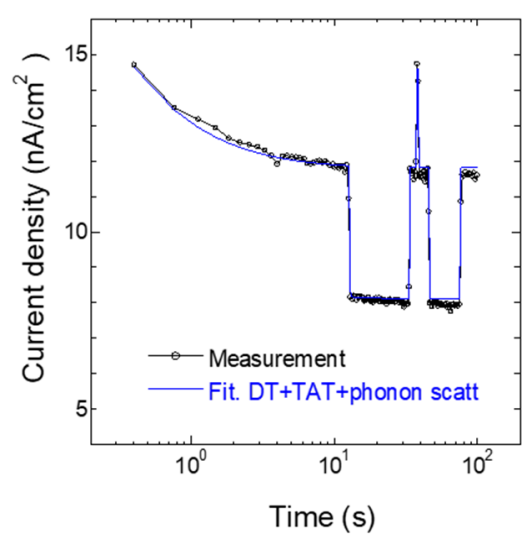

(d)

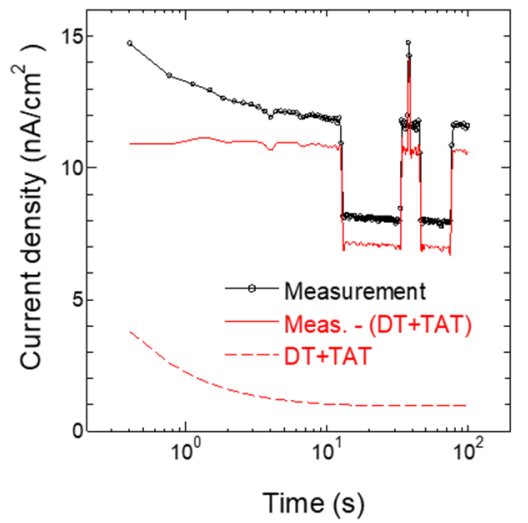

(b)

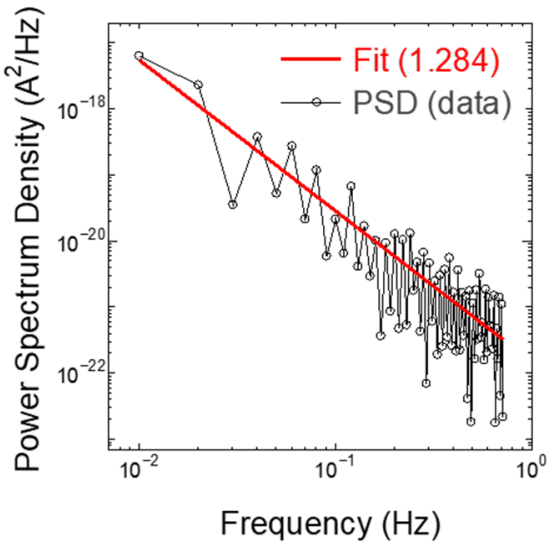

(e)
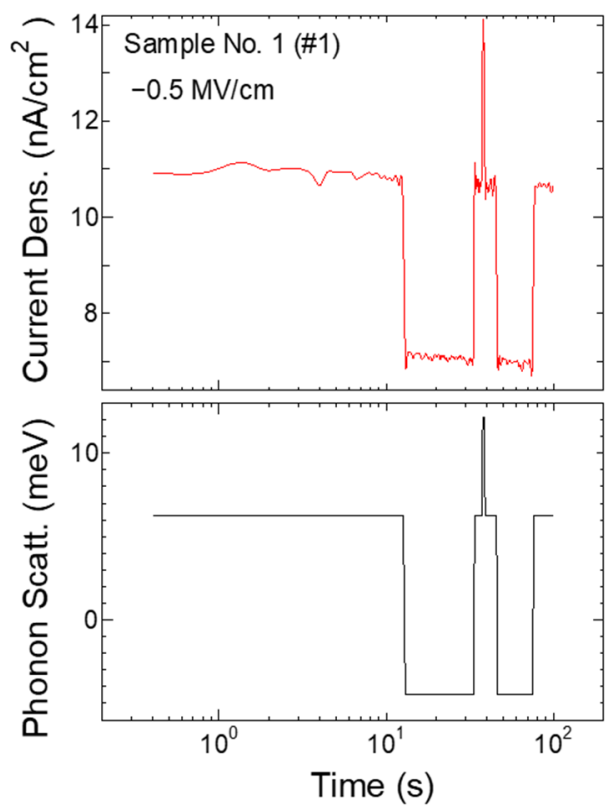

(c)

Figure 18. Fit uneven fluctuation with sample No. 1 (\#1) under $-0.5 \mathrm{MV} / \mathrm{cm}$. See Figure 8 in $[30]$. In (a,b,d) and the upper of (c), the vertical axis is the current density and the horizontal one is time. In the bottom of (c), the vertical axis is phonon scattering energy and the horizontal one is time. In (e), the vertical axis is the power spectrum density and the horizontal one is the frequency. For the calibration of parameters, we synchronized the occurrence of discrete perturbations and phonon scattering. The marks in (e) are converted from measured transient leakage current density using (6a). By fitting the marks, we could extract $\alpha$ using (6b).

Table 2. Calibrated fitting parameters with uneven fluctuation. See Table 2 in [30]. The trap-assisted tunneling (TAT) can be involved in a cell and not in another cell at the same electric field. See $\# 1$ and \#3 at $-0.5 \mathrm{MV} / \mathrm{cm}$. In \#1, the TAT is involved, but not in \#3. It may be observed that the trap levels of the interface layer are lower in \#1 than in \#3.

\begin{tabular}{cccccccccc}
\hline Sample No. & $\begin{array}{c}\text { Field } \\
(\mathbf{M V} / \mathbf{c m})\end{array}$ & $\begin{array}{c}J_{g, \infty} \\
\left(\mathbf{p A} / \mathbf{c m}^{2}\right)\end{array}$ & $\boldsymbol{\tau}(\mathbf{s})$ & $\boldsymbol{s}$ & $\begin{array}{c}\boldsymbol{J}_{\mathbf{g}, \boldsymbol{\infty}}^{\prime} \\
\left(\mathbf{p A} / \mathbf{c m}^{\mathbf{2}}\right)\end{array}$ & $\boldsymbol{\tau}^{\prime}(\mathbf{s})$ & $\boldsymbol{s}^{\prime}$ & Note & $\boldsymbol{\alpha}$ \\
\hline$\# 1$ & -0.5 & 160 & 9.6 & 0.9880 & 798 & 0.1 & 0.1 & DT + TAT & 1.284 \\
\hline$\# 3$ & -0.5 & 25.5 & 74.6 & 0.9950 & $\mathrm{~N} / \mathrm{A}$ & $\mathrm{N} / \mathrm{A}$ & $\mathrm{N} / \mathrm{A}$ & $\mathrm{DT}$ & 1.322 \\
\hline$\# 4$ & 1.0 & 114 & 50 & 0.9950 & 343 & 0.1 & 0.1 & DT + TAT & 1.289 \\
\hline$\# 7$ & 0.7 & 184 & 21.8 & 0.9870 & 2020 & 1 & 0.8 & DT + TAT & 0.927 \\
\hline$\# 8$ & -0.7 & 47.1 & 85 & 0.9960 & 588 & 12 & 0.2 & DT + TAT & 1.367 \\
\hline
\end{tabular}

Let us remember that multiple grains of the $10 \mathrm{~nm} \mathrm{ZrO}_{2}$ may face either of the cathode or anode interfaces. Accordingly, there may be grain boundary patterns at the interfaces. Some traps are on the grain boundaries and others are out of the grain boundaries at the interfaces. We can suppose that the details of trap states are more complicated on the grain boundaries. This may cause the barrier height fluctuations $\left(\Delta \varphi_{c}\right.$ and $\left.\Delta \varphi_{a}\right)$ in the cathode and anode interfaces, respectively. The electrons captured by the trap sites on the grain 
boundaries may change the trap states by emitting and absorbing the phonon energy $\left(\Delta E_{p}\right)$. For the phonon absorption, $\Delta E_{p}$ is positive. For the phonon emission, $\Delta E_{p}$ is negative. Thus, the dwell time of electrons stored in the cathode interface may fluctuate, as follows: where the suffixes of \pm depict the positive and negative electric fields, respectively, and $\tau_{ \pm 0}$ is the dwell time without the uneven component.

$$
\tau_{ \pm}=\tau_{ \pm 0} \exp \left(\frac{-\Delta \varphi_{c}+\Delta E_{\mathrm{p}}}{\Delta_{c}}+\frac{-\Delta \varphi_{a}+\Delta E_{\mathrm{p}}}{\Delta_{a}}\right)
$$

The denominators of the terms in the exponent of (9) are written as follows: where $m^{*}$ is the effective tunnel mass and assumed to be half the rest of the electron mass, $t_{I}$ is the thickness of the insulator (10 $\mathrm{nm}$ in this case), $E C_{c}$ and $E C_{a}$ are the conduction band edges in the cathode and anode interfaces, respectively, and $u^{*}$ is the compensation of $u$.

$$
\Delta_{u}=-\frac{3}{2} \frac{\hbar}{\sqrt{m^{*}}} \frac{1}{t_{I}} \frac{\left(E C_{u}-E C_{u^{*}}\right)^{2}}{E C_{u}^{3 / 2}-3 E C_{u}^{1 / 2} E C_{u^{*}}+E C_{u^{*}}^{3 / 2}}
$$

If $u^{*}=a$, then $u=c$ and vice versa. In the bottom plot of Figure 18c, by using these parameters related to phonon scattering, we can extract $\Delta E_{p}$ in the vertical axis over time in the horizontal axis. The upper is the replot of the uneven component depicted by the red line in (b). However, for the calibration of parameters in (9) and (10), we synchronized the occurrence of phonon scattering in our model and that of discrete perturbations in the extracted uneven component (i.e., in the measurement). In (d) of this figure, we obtained an excellent agreement with the measurement by using (7)-(10) with the phonon scattering (i.e., RTN), as depicted by the blue line. The calibrated parameters for phonon scattering are shown in Table 3 (see \#1 therein). In (e), furthermore, we can convert this measurement to the power spectrum density in the frequency domain using (6a). We can thus extract $\alpha=1.284$ using $(6 b)$.

Table 3. Calibrated parameters related to phonon scattering. See Table 3 in [30]. The barrier height fluctuation varies over samples around $100 \mathrm{meV}$.

\begin{tabular}{cccccc}
\hline Sample No. & $\begin{array}{c}\text { Field } \\
(\mathbf{M V} / \mathbf{c m})\end{array}$ & $\begin{array}{c}\Delta \varphi_{c} \\
(\mathbf{m e V})\end{array}$ & $\begin{array}{c}\Delta \varphi_{a} \\
(\mathbf{m e V})\end{array}$ & $\Delta_{c}$ & $\Delta_{a}$ \\
\hline$\# 1$ & -0.5 & 58.22 & 104.74 & 29.47 & 206.57 \\
\hline$\# 3$ & -0.5 & 109.22 & 74.6 & 29.47 & 206.57 \\
\hline$\# 4$ & 1.0 & 153.87 & 185.41 & 30.59 & 179.50 \\
\hline$\# 7$ & 0.7 & 85.59 & 143.39 & 29.73 & 205.42 \\
\hline$\# 8$ & -0.7 & 134.90 & 119.21 & 29.73 & 205.42 \\
\hline
\end{tabular}

We also observed an uneven transient current with samples \#3 at $-0.5 \mathrm{MV} / \mathrm{cm}, \# 4$ at $+1.0 \mathrm{MV} / \mathrm{cm}, \# 7$ at $+0.7 \mathrm{MV} / \mathrm{cm}$, and \#8 at $-0.7 \mathrm{MV} / \mathrm{cm}$. In a similar manner, using (7)-(10), we obtained an excellent agreement for the measured transient current densities, as shown in Figure 19. The vertical axis is current density and the horizontal one is time. In (a), we plot the fitting results of $\# 1$ at $-0.5 \mathrm{MV} / \mathrm{cm}$ (replot of Figure $18 \mathrm{~d}$ ), $\# 3$ at $-0.5 \mathrm{MV} / \mathrm{cm}$, and $\# 4$ at $+1.0 \mathrm{MV} / \mathrm{cm}$. In (b), we plot the fitting result of $\# 7$ at $+0.7 \mathrm{MV} / \mathrm{cm}$ and $\# 8$ at $-0.7 \mathrm{MV} / \mathrm{cm}$. The calibrated parameters are shown in Tables 2 and 3. Subsequently, using the same method to obtain Figure 18c, we extracted phonon energies using these samples, as shown in Figure 20. In the upper plots, we show the discrete perturbation (i.e., uneven component) over time. In the bottom plot, we show the extracted $\Delta E_{p}$ over time. However, for the calibration of parameters in (9) and (10), we synchronized the occurrence of discrete electronic perturbations and phonon scattering. The $\alpha$ extracted using the same method to obtain Figure 18e was around 1.0, as shown in Table 2. Thus, we can find that phonon scattering generates randomness due to the $1 / f^{2}$-fluctuation, that is, Brownian noise. 


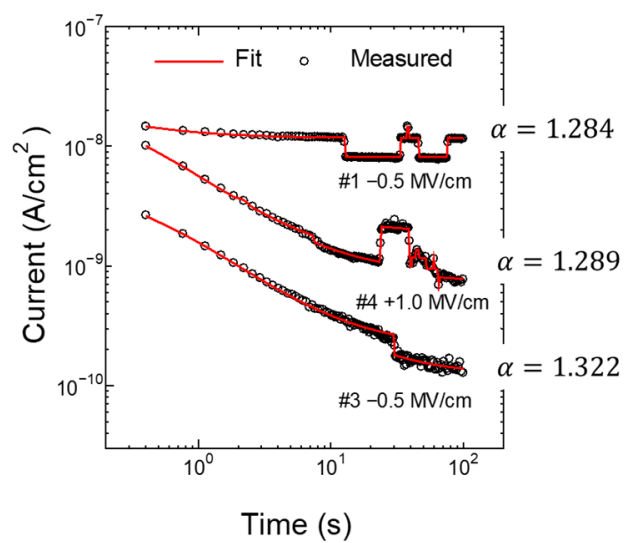

(a)

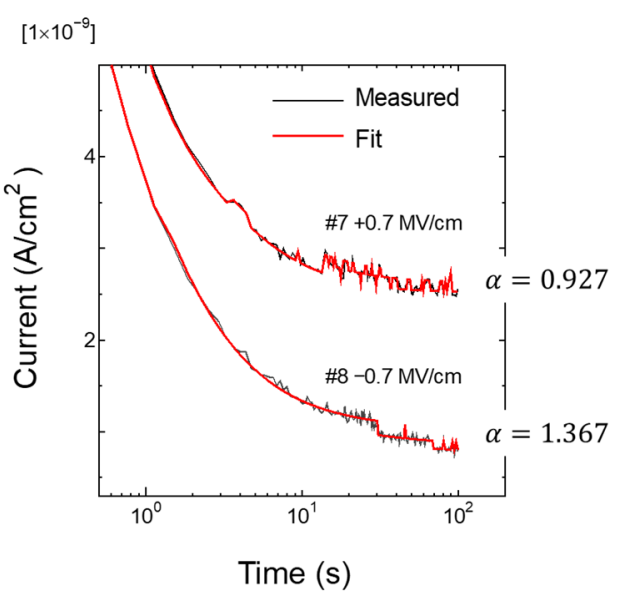

(b)

Figure 19. Fit uneven fluctuations. See Figures 8 and 9 in [30]. The vertical axis is the current density and the horizontal one is time. In (a), we compare the measured and calculated data for samples $\# 1$ at $-0.5 \mathrm{MV} / \mathrm{cm}, \# 4$ at $+1.0 \mathrm{MV} / \mathrm{cm}, \# 3$ at $-0.5 \mathrm{MV} / \mathrm{cm}$. In (b), we compare the measured and calculated data for samples \#7 at $+0.7 \mathrm{MV} / \mathrm{cm}$, and \#8 at $-0.7 \mathrm{MV} / \mathrm{cm}$. Excellent agreement was obtained using (7)-(10) and the calibrated parameters in Tables 2 and 3. The extracted $\alpha$ was 1.284, $1.289,1.322,0.927$, and 1.367 for samples \#1 at $-0.5 \mathrm{MV} / \mathrm{cm}$, \#4 at $+1.0 \mathrm{MV} / \mathrm{cm}, \# 3$ at $-0.5 \mathrm{MV} / \mathrm{cm}$, $\# 7$ at $+0.7 \mathrm{MV} / \mathrm{cm}$, and \#8 at $-0.7 \mathrm{MV} / \mathrm{cm}$, respectively.

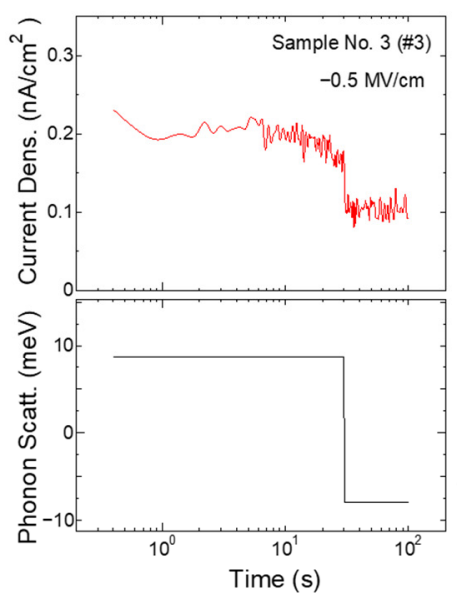

(a)

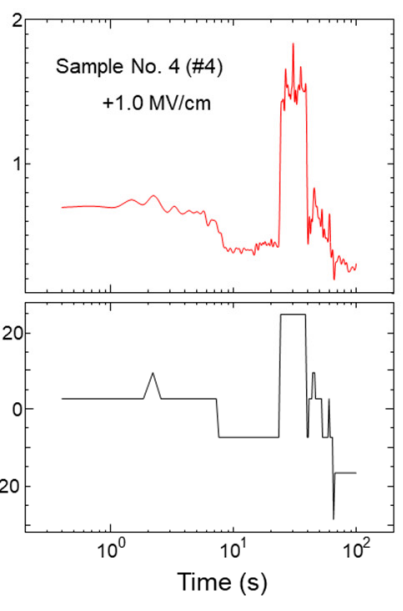

(b)

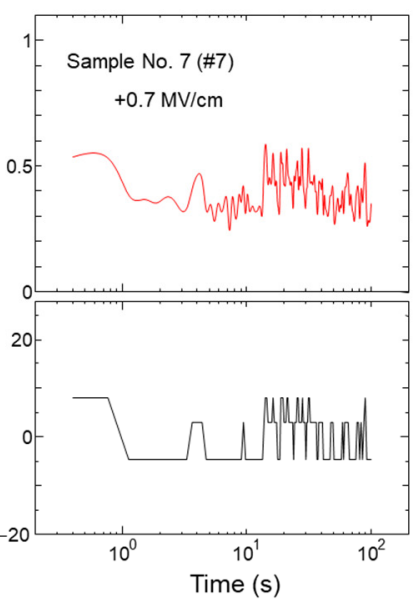

(c)

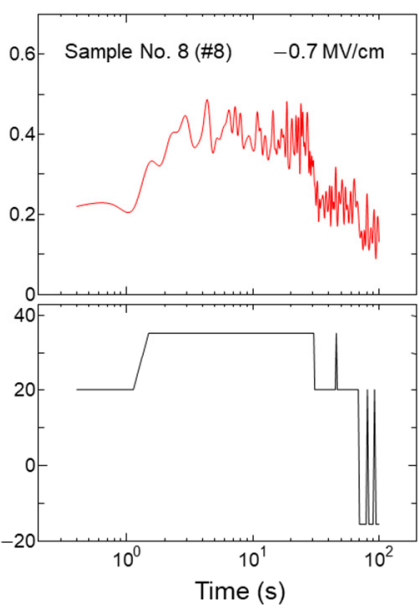

(d)

Figure 20. Extracted phonon energies. See Figure 10 in [30]. In the upper plots, the vertical axis is the uneven component of measured transient current density (discrete electronic perturbations) and the horizontal one is time. In the bottom plots, the vertical axis is phonon scattering energies and the horizontal one is time. (a-d) for the samples \#3 at $-0.5 \mathrm{MV} / \mathrm{cm}$, \#4 at $+1.0 \mathrm{MV} / \mathrm{cm}, \# 7$ at $+0.7 \mathrm{MV} / \mathrm{cm}$, and \#8 at $-0.7 \mathrm{MV} / \mathrm{cm}$, respectively. For the calibration of parameters, we synchronized the occurrence of discrete electronic perturbations and phonon scattering.

By comparing with the theoretical infrared phonon scattering [43], we can suppose that if $\Delta E_{p}>10 \mathrm{meV}$ then grains are cubic or tetragonal, which is consistent with the $10 \mathrm{~nm} \mathrm{ZrO}_{2}$ of the samples used in the present measurement. See Figure 10b. Otherwise, they are monoclinic grains. In this way, we can deduce some physical intuitions from the phonon scattering analysis. By repeating the measurement of the same samples with the same conditions, sometimes we could obtain such uneven fluctuations. On other occasions, we could not obtain it since the discrete electronic perturbations were unstable, as we have shown in Figures 4-7. 
However, it may be still an open problem how phonons scatter with a discrete local trap. Grasser et al. reported a time-dependent analysis of metastable defect states [44] and Ielmini et al. performed a structure relaxation due to trapped holes [45]. It would be interesting to know how or if such details are related to phonon scattering, as we could extract some more detailed physical intuitions from phonon scattering energies.

Nowadays, there are many kinds of semiconductor memory. As conventional ones with MIM capacitors, we have static random-access memory (SRAM), dynamic randomaccess memory (DRAM), NOR flash, and NAND flash. As emerging types, we have phasechange random access memory (PCRAM), magnetic random access memory (MRAM), resistivity random access memory (RRAM), and so forth. The analysis of spectral index will be critically important for investigating various kinds of reliability issues, not only in the conventional ones, but also in the emerging ones. For example, a complex RTN was reported in RRAM [23,46].

\section{Conclusions}

We consider that sample variation is attributable to grain distributions in stacking oxide films. We thus proposed the formulae to eliminate sample variations from measured transient gate leakage current flowing through stacking oxide films. Thanks to this, we could analyze the discrete electronic perturbations which are seen in measured transient gate leakage current.

The transient gate leakage current has unstable discrete electronic perturbations that are related to local traps existing at the interfaces of the gate stack and inside. By repeatedly measuring the same samples with the same conditions, sometimes we could observe this, but not on other occasions. By assuming that the emission from traps is a stationary process, power spectrum analysis shows that the direct tunneling is subject to the $1 / f$-fluctuation, that is, the Flicker noise. However, the trap-assistance and the interface roughness break the power law of the $1 / f$-fluctuation. The random telegraph noise due to phonon-scattering shows a $1 / f^{2}$-fluctuation, that is the Brownian noise. Even though it is hard to observe a local trap by physical inspection, we may have a chance to characterize it by using transient analysis.

Funding: The second half of this research was funded by Tokyo Electron Ltd. (Tokyo, Japan).

Acknowledgments: The second half of the present work was performed with respect to the joint development agreement between Tokyo Electron Ltd., and the National Yang Ming Chiao Tung University [30]. The authors would like to thank K. Akiyama, Y. Hirota, Y. Akasaka, G. Nakamura, H. Nagai, T. Morimoto, Y. Morozumi, S. Hsihiya, F. Hayase, K. Harada. The first half of the present work was performed with T. Ushijima, N. Hagiwara, C. Okada, and T. Tanabe [8]. National Chiao Tung University was combined with National Yang Ming University to be National Yang Ming Chiao Tung University since 1 February 2021.

Conflicts of Interest: The authors declare no conflict of interest.

\section{References}

1. Dennard, R.H.; Gaensslen, F.H.; Yu, H.-N.; Rideout, V.L.; Bassous, E.; LeBlanc, A.R. Design of ion-implanted MOSFET's with very small physical dimensions. IEEE J. Solid-State Sicruits 1974, 9, 256-268.

2. Masanet, E.; Shhehabi, A.; Lei, N.; Smith, S.; Koomey, J. Recalibrating global data center energy-use estimates. Science 2020, 367, 984-986. [CrossRef]

3. Moras, G.; Robayo, D.A.; Lopez, J.M.; Grenouillet, L.; Carabasse, C.; Navarro, G.; Sabbione, C.; Bernard, M.; Cagli, C.; Castellani, N.; et al. Crosspoint memory arrays: Principle, strengths and challenges. In Proceedings of the 2020 IEEE International Memory Workshop, Dresden, Germany, 17-20 May 2020.

4. Dierling, K.; Das, M. GPUs, DPUs, and Storage: Bringing AI and ML to data centers everywhere. In Proceedings of the Flash Memory Summit 2020, Santa Clara, CA, USA, 10-12 November 2020.

5. Ilkbahar, A. Intel ${ }^{\circledR 囚}$ Optane ${ }^{\mathrm{TM}}$ Persistent Memory from vision to reality. In Proceedings of the Flash Memory Summit 2020, Santa Clara, CA, USA, 10-12 November 2020.

6. Burstein, E.; Lundqvist, S. (Eds.) Tunneling Phenomena in Solids; Lectures Presented at the 1967 NATO Advanced Study Institute at RiO, Denmark; Plenum: New York, NY, USA, 1969; p. 35. 
7. Watanabe, H. Tutorial: Trap-related reliability issues in NAND Flash memory. In Proceedings of the IEEE International Reliability Physics Symposium, Anaheim, CA, USA, 15-19 April 2012.

8. Watanabe, H.; Ushijima, T.; Hagiwara, N.; Okada, C.; Tanabe, T. Integrated Batteryless Electron Timer. IEEE Trans. Electron Devices 2010, 58, 792-797. [CrossRef]

9. Marichal, O.; Wybo, G.; van Camp, B.; Vanysacker, P.; Keppens, B. SCR-based ESD protection in nanomerter SOI technologies. Microelectron. Reliab. 2007, 47, 1060-1068. [CrossRef]

10. Naruke, K.; Taguchi, S.; Wada, M. Stress induced leakage current limiting to scale down EEPROM tunnel oxide thickness. In Proceedings of the Technical Digest of the 1988 IEEE International Electron Devices Meeting, San Francisco, CA, USA, 11-14 December 1988; pp. 424-427.

11. Ielmini, D.; Spinelli, A.S.; Lacaita, A.L.; Modelli, A. A new two-trap tunneling model for the anomalous stress-induced leakage current (SILC) in Flash memories. Microelectron. Eng. 2001, 59, 189-195. [CrossRef]

12. Inatsuka, T.; Kumagai, Y.; Kuroda, R.; Teramoto, A.; Suwa, T.; Ohmi, S.S.T. A test circuit for extremely low gate leakage current measurement of 10 aA for 80,000 MOSFETs in 80s. IEEE Trans. Semicond. Manuf. 2013, 26, 288. [CrossRef]

13. Köcher, A.; Tohoku University in those days. Hiroshima University at present, Sendai, Japan. Personal communication, 2012.

14. Watanabe, H.; Matsushita, D.; Muraoka, K.; Kato, K. Universal tunnel mass and charge trapping in $\left[\left(\mathrm{SiO}_{2}\right)_{1-\mathrm{x}}\left(\mathrm{Si}_{3} \mathrm{~N}_{4}\right) \mathrm{x}\right]_{1-\mathrm{y}} \mathrm{Siy}$ film. IEEE Trans. Electron Devices 2010, 57, 1129-1136. [CrossRef]

15. Simmons, J.G. Poole-Frenkel Effect and Schottky Effect in Metal-Insulator-Metal Systems. Phys. Rev. 1967, 155, 657-660. [CrossRef]

16. Takagi, S.; Yasuda, N.; Toriumi, A. Experimental evidence of inelastic tunneling and new I-V model for stress-induced leakage current. In Proceedings of the Technical Digest of the 1996 IEEE International Electron Devices Meeting, San Francisco, CA, USA, 8-11 December 1996; pp. 323-326.

17. Wu, J.; Register, L.F.; Rosenbaum, E. Trap-assisted tunneling current through ultra-thin oxide. In Proceedings of the 37th IEEE International Reliability Physics Symposium, San Diego, CA, USA, 23-25 March 1999; pp. 389-395.

18. Machlup, S. Noise in semiconductors: Spectrum of a two-parameter random signal. J. Appl. Phys. 1954, 25, 341-343. [CrossRef]

19. Dutta, P.; Horn, P.M. Low-frequency fluctuations in solids: 1/f-noise. Rev. Mod. Phys. 1981, 53, 497-516. [CrossRef]

20. Bak, P.; Tang, C.; Wiesenfeld, K. Self-organized criticality: An explanation of the 1/f noise. Phys. Rev. Lett. 1987, 59, 381-384. [CrossRef]

21. Weissman, M.B. 1/f noise and other slow, nonexponential kinetics in condensed matter. Rev. Mod. Phys. 1988, 60, 537-571. [CrossRef]

22. Kogan, S. Electronic Noise and Fluctuations in Solids; England Cambridge University Press: Cambridge, UK, 1996; pp. 205-208.

23. Raghavan, N.; Degraeve, R.; Fantini, A.; Goux, L.; Strangio, S.; Govoreanu, B.; Wouters, D.J.; Groeseneken, G.; Jurczak, M. Microscopic origin of random telegraph noise fluctuations in aggressively scaled RRAM and its impact on read disturb variability. In Proceedings of the IEEE IRPS, Monterey, CA, USA, 14-18 April 2013.

24. McWhorter, A.L. Semiconductor Surface Physics; Kingston, R.H., Ed.; University of Pennsylvania Press: Philadelphia, PA, USA, 1957.

25. Christensson, S.; Lundström, I.; Svensson, C. Low frequency noise in MOS transistors-I. theory. Solid-State Electron. 1968, 11, 797-812. [CrossRef]

26. Christensson, S.; Lundström, I. Low frequency noise in MOS transistors-II. Experiments. Solid-State Electron. 1968, 11, 813-820. [CrossRef]

27. Uren, M.J.; Day, D.J.; Kirton, M.J. $1 / f$ and random telegraph noise in silicon metal-oxide-semiconductor field-effect transistors. Appl. Phys. Lett. 1985, 47, 1195. [CrossRef]

28. Kirton, M.J.; Uren, M.J. Noise in solid-state microstructures: A new perspective on individual defects, interface states and low frequency $(1 / f)$ noise. Adv. Phys. 1989, 38, 367. [CrossRef]

29. Boyat, A.; Joshi, B. A review paper: Noise models in digital image processing. Signal Image Process. Int. J. 2015, 6, 63-75. [CrossRef]

30. Lin, H.-J.; Akiyama, K.; Hirota, Y.; Akasaka, Y.; Nakaumura, G.; Nagai, H.; Morimoto, T.; Watanabe, H. Experimental study of $1 / f^{1+\alpha}$ noise in transient leakage current of metal-insulator-metal with stacked high-K polycrystalline films. IEEE Trans. Electron Devices 2020, 67, 2503-2509. [CrossRef]

31. Robertson, J. Band offsets of high dielectric constant gate oxides on silicon. J. Non-Cryst. Solids 2002, 303, 994-1000. [CrossRef]

32. Didden, A.; Battjes, H.; Machunze, R.; Dan, B.; van de Krol, R. Titanium nitride: A new ohmic contact material for n-type CdS. J. Appl. Phys. 2011, 110, 033717. [CrossRef]

33. Williams, R. Photoemission of electrons from silicon to silicon dioxide. Phys. Rev. 1965, 140, A569-A575. [CrossRef]

34. Watanabe, H. Transient device simulation of floating gate nonvolatile memory cell with a local trap. IEEE Trans. Electron Devices 2010, 57, 1873-1882. [CrossRef]

35. Chang, C.M.; Chung, S.S.; Hsieh, Y.S.; Cheng, L.W.; Tsai, C.T.; Ma, G.H.; Chien, S.C.; Sun, S.W. The Observation of Trapping and Detrapping Effects in High-k Gate Dielectric MOSFETs by a New Gate Current Random Telegraph Noise (IG-RTN) Approach. In Proceedings of the Technical Digest of the 2008 IEEE International Electron Devices Meeting, San Francisco, CA, USA, 15-17 December 2008.

36. Hooge, F.N. 1/f noise is no surface effect. Phys. Lett. A 1969, 29, 139-140. [CrossRef]

37. Hooge, F.N. Discussion of recent experiments on 1/f noise. Physica 1976, 60, 130-144. [CrossRef]

38. Hooge, F.N.; Vandamme, L.K.J. Lattice scattering causes 1/f noise. Phys. Lett. A 1978, 66, 315-316. [CrossRef] 
39. Hung, K.K.; Ko, P.K.; Hu, C.; Cheng, Y.C. A unified model for the flicker noise in metal-oxide-semiconductor field-effect transistors. IEEE Trans. Electron Devices 1990, 37, 654-665. [CrossRef]

40. Hung, K.K.; Ko, P.K.; Hu, C.; Cheng, Y.C. A physics-based MOSFET noise model for circuit simulators. IEEE Trans. Electron Devices 1990, 37, 1323-1333. [CrossRef]

41. Hung, K.K.; Ko, P.K.; Hu, C.; Cheng, Y.C. Random telegraph noise of deep-submicrometer MOSFETs. IEEE Electron Device Lett. 1990, 11, 90-92. [CrossRef]

42. Jindal, R.P.; van der Ziel, A. Carrier fluctuation noise in a MOSFET channel due to traps in the oxide. Solid-State Electron. 1978, 21, 901-903. [CrossRef]

43. Zhao, X.; Vnanderbilt, D. Phonons and lattice dielectric properties of zirconia. Phys. Rev. B Condens. Matter 2002, 65, 075105. [CrossRef]

44. Grasser, T.; Reisinger, H.; Wagner, P.-J.; Schanovsky, F.; Goes, W.; Kaczer, B. The time dependent defect spectroscopy (TDDS) for the characterization of the bias temperature instability. In Proceedings of the IEEE International Reliability Physics Symposium, Anaheim, CA, USA, 2-6 May 2010; pp. 16-25.

45. Ielmini, D.; Manigrasso, M.; Gattel, F.; Valentini, M.G. A new NBTI model based on hole trapping and structural relaxation in MOS dielectrics. IEEE Trans. Electron Devices 2009, 56, 1943-1952. [CrossRef]

46. Ambrogio, S.; Balatti, S.; Cubeta, A.; Calderoni, A.; Ramaswamy, N.; Ielmini, D. Understanding switching variability and random telegraph noise in resistive RAM. In Proceedings of the Technical Digest of the 2013 IEEE International Electron Devices Meeting, Washington, DC, USA, 9-11 December 2013; pp. 782-785. 